\title{
Polar-Interball coordinated observations of plasma and magnetic field characteristics in the regions of the northern and southern distant cusps
}

\author{
E. Dubinin, ${ }^{1,2}$ A. Skalsky, ${ }^{3}$ P. Song, ${ }^{1}$ S. Savin, ${ }^{3}$ J. Kozyra, ${ }^{1}$ T. E. Moore, ${ }^{4}$ \\ C. T. Russell, ${ }^{5}$ M. O. Chandler, ${ }^{6}$ A. Fedorov, ${ }^{3}$ L. Avanov, ${ }^{3}$
}

J.-A. Sauvaud, ${ }^{7}$ and R. H. W. Friedel ${ }^{8}$

Received 7 December 2000; revised 23 March 2001; accepted 23 April 2001; published 14 May 2002.

[1] The structure of the high-latitude magnetosphere near the polar cusps is studied by using coordinated observations from the Polar and Interball satellites. While Polar surveyed the northern polar cusp region, Interball sampled the high-altitude region in the vicinity of the southern cusp in similar local times. A "magnetic turbulent region" that is distinct from the magnetosphere and magnetosheath and consists of recurrent small-scale events is observed in both hemispheres. The small-scale events often contain a steep ramp followed by a wave train. The events are characterized by a steep increase in the ion energy followed by dispersed velocity filter features. Kinetic and fluid characteristics of ions and a test of the Walen relation show that the events are associated with reconnection processes. S-shaped polarization of the magnetic field variations is a characteristic feature in many cases indicating the existence of intermediate shocks. The change in the low-energy cutoff of injected ions indicates that the reconnection site is about a few Earth radii from Polar. Reflected ions on the reconnected magnetospheric field lines, which are not predicted by simple reconnection scenarios, are also observed. Coexistence of transmitted and reflected ions gives rise to a "stagnant" plasma on the magnetospheric field lines. Characteristics of ions and electrons strongly support the suggestion that the field lines threading the plasma in these regions, which extend from the magnetosheath to the magnetosphere, are connected, at least at one end, with the Earth. In other words, the boundary layer is located, at least partly, on reconnected field lines. In contrast, a turbulent region in the indentation of the cusps has been thought to be in the magnetosheath and not to be connected with the Earth. Although the events observed by both satellites have a certain similarity, the probability of conjugated events being recorded by both satellites is small. It is believed that the visible similarity between the observations from the two hemispheres is due to structures that are characteristic features of a single transient reconnection event. INDEX TERMS: 2724 Magnetospheric Physics: Magnetopause, cusp, and boundary layers; 7835 Space Plasma Physics: Magnetic reconnection; 2784 Magnetospheric Physics: Solar wind/ magnetosphere interactions

\section{Introduction}

[2] It is commonly believed that the polar cusps play an essential role in the entry of the solar wind into the Earth's magnetosphere although there is an ongoing debate over what exactly the cusps are. The concept of the cusps originates from the

\footnotetext{
${ }^{1}$ Space Physics Research Laboratory, University of Michigan, Ann Arbor, Michigan, USA.

${ }^{2}$ Now at Max-Planck-Institut für Aeronomie, Katlenburg-Lindau, Germany.

${ }^{3}$ Space Research Institute, Moscow, Russia.

${ }^{4}$ Laboratory for Extraterrestrial Physics, Goddard Space Flight Center, Greenbelt, Maryland, USA.

${ }^{5}$ Institute of Geophysics and Planetary Physics, University of California, Los Angeles, California, USA.

${ }^{6}$ Space Science Laboratory, NASA Space Flight Center, Huntsville, Alabama, USA.

${ }^{7}$ Centre d'Etude Spatiale des Rayonnements-Centre National de la Recherche Scientifique, Toulouse, France.

${ }^{8}$ Los Alamos National Laboratory, Los Alamos, New Mexico, USA.

Copyright 2002 by the American Geophysical Union.

0148-0227/02/2001JA900068
}

theoretical view of a separatrix between the dayside and nightside magnetic field lines. Singularities at the cusps lead to a mapping of the entire magnetopause to narrow regions near local noon through which intense precipitation of the magnetosheath plasma occurs. Observationally, the cusp was at first detected at low and intermediate altitudes by Heikkila and Winningham [1971] and Frank [1971]. Laboratory modeling experiments have also shown evidence of the direct entry of plasma through the cusps [Dubinin et al., 1971; Dubinin and Podgorny, 1974]. The broader region of magnetosheath plasma precipitation in the vicinity of the cusp was termed the "cleft" [Heikkila and Winningham, 1971]. Later, the cleft was envisaged as being mapped from the low-latitude boundary layer [Newell and Meng, 1988; Lundin, 1988]. The cusp/cleft has always been treated as a key to understanding of mass and momentum transfer into the Earth's magnetosphere. The main plasma characteristics of high-altitude cusps were obtained by HEOS-2 and Prognoz-7 satellites in the 1970s and 1980s [Paschmann et al., 1976; Haerendel and Paschmann, 1975; Lundin, 1985]. The HEOS-2 measurements in the polar cusp reveal the existence of a plasma layer typically $1-2 R_{\mathrm{E}}$ thick just inside the magnetopause. The layer was thought to be a consequence of the entry of magnetosheath plasma and was referred to as the "entry layer" [Haerendel and Paschmann, 1975; Pasch- 
mann et al., 1976]. The main feature of this layer is disordered plasma flow, in contrast to the laminar flow in the adjacent magnetosheath and plasma mantle. According to their definition the polar cusp comprises three main regions: (1) the region of stagnant plasma in the adjacent magnetosheath, (2) the entry layer, and (3) the mantle region of the cusp. However, the low data rates of the HEOS-2 satellite could not resolve the detailed structures in this critical region.

[3] It is also believed that the phenomena near the cusp are strongly controlled by the orientation of the interplanetary magnetic field (IMF). Analysis of HEOS-2 data indicated that reconnection sites were near the cusp regions [Haerendel et al., 1978]. The laboratory simulation experiments have also shown that the cusps are the most favorable sites for reconnection [Dubinin et al., 1978]. The concept of antiparallel merging proposed by Crooker [1979] assumed that reconnection occurs where the magnetosheath magnetic field is antiparallel to the Earth's magnetic field. In the regions of the magnetosphere with a cusp-like geometry, antiparallel fields occur near the cusps for virtually all orientations of the IMF. Reconnection processes in the near-cusp regions may essentially determine the global structure of the magnetopause-cusp interface. Moreover, it is not clear at present how to identify the magnetopause in the near-cusp region because of the complexity of the current system there and the ambiguity in different possible interpretations of the same observed signatures.

[4] The Polar and Interball observations at high altitudes may shed new light on these questions. The recent observations made by Interball show that for steady southward IMF with a large $B_{y}$ component the entry of magnetosheath plasma into the magnetosphere can be well described in terms of reconnection [Fedorov et al., 2000]. The observations made by Polar also provided new evidence of magnetic merging near the cusps for northward IMF, although some controversy remains [Chandler et al., 1999; Russell et al., 2000]. Just outside of the high-latitude magnetopause, a turbulent boundary layer was identified [Savin et al., 1998]. The region contains highly structured magnetic field and accelerated/ heated magnetosheath plasma flows, both of which could be interpreted as small-scale reconnection events. Although supporting evidence for reconnection at the low-latitude magnetopause [Paschmann et al., 1979; Sonnerup et al., 1981; Gosling et al., 1991; Fuselier et al., 1995] is abundant (as summarized by Song et al. [1995]), the knowledge about reconnection in the cusp/magnetopause region is limited. The reconnection processes can be quasisteady state and/or transient [Russell and Elphic, 1978]. Transient reconnection phenomena have not been studied from in situ observations at high latitudes. It is worth noting that transient phenomena can also be driven by solar wind pressure variations. Both processes, reconnection and Kelvin-Helmholz instability, operate at the low-latitude magnetopause. There has been heated debate over whether these processes can be distinguished.

[5] In this paper, we study the structure of the high-latitude magnetopause-cusp interface using correlated observations made by the Polar and Interball satellites. While Polar surveyed the northern polar cusp region, Interball sampled the high-altitude regions in the vicinity of the southern cusp. The phenomena in the magnetosheath-magnetopause-cusp interface are complicated, and it is often difficult to identify whether a satellite is in the sheath or inside the magnetosphere. The magnetospheric field is often easy to identify because of its smooth variations (lack of substantial small-scale fluctuations). The fluctuations in the cusp and the boundary layer may have similar field characteristics and are difficult to distinguish from the magnetosheath field. Rapid motion of the magnetopause and the bow shock in response to upstream variations can cause a satellite to move rapidly across the boundaries and further complicate the problem. To resolve this ambiguity, Song et al. [1992] proposed to correlate the clock angles in interplanetary space and the magnetosheath. Song et al. [1992] have shown that the clock angle does not change significantly from the solar wind to the magnetosheath. Correlation between the clock angles in the interplanetary space and the magnetosheath provides a good indicator of the sheath. Recently, Song et al. [1999a, 1999b] have developed a more sophisticated scheme to resolve the spatialtemporal ambiguity. They used the gas dynamic convected field (GDCF) model [Spreiter and Stahara, 1980] to study dynamic solar wind-magnetopause interaction. The solar wind measurements from an upstream monitor are input into the GDCF model, and then the model output is compared with in situ measurements in the magnetosheath/boundary layer. This method provides oneto-one correlation of the solar wind and magnetosheath measurements as well as the sheath satellite location with respect to the magnetopause boundary. In this paper, we use both techniques to distinguish the sheath region from the boundary layers and then to study the particle characteristics in each region.

\section{Instruments}

[6] Interball and Polar represent a set of satellites developed for a comprehensive study of physical processes at the high-latitude magnetosphere. The Polar spacecraft was launched in February 1996 , onto a $90^{\circ}$ inclination polar elliptical orbit $\left(2 \times 9 R_{E}\right)$. Polar does not observe the magnetopause and magnetosheath under normal solar conditions, but in periods of high solar wind dynamic pressure it crosses the magnetopause/exterior cusp. In this paper we will focus on one such occasion. Interball, launched into an orbit with an apogee near $31 R_{E}$ and inclination $\sim 65^{\circ}$, probes the main magnetospheric regions including the high-latitude magnetosheath-cusp interface. Experiments used in this study are the magnetometers on both satellites and Polar Thermal Ion Dynamics Experiment (TIDE), Interball CORALL, and Interball ELECTRON plasma spectrometers. The TIDE on the Polar spacecraft provides measurements of ions in the energy range from 0.3 to 500 $\mathrm{eV}$ [Moore et al., 1995]. Although TIDE was originally designed to discriminate between ion species, a failure of the mass sensor occurred prior to this study event. As a result, no mass-resolved information is available. The measurements from different elevation angles are transmitted as a single signal during the study event. Therefore the TIDE measurements can be treated as from a wideangle detector similar to the ISEE Fast Plasma Experiment. The energy and angular resolutions in the azimuth are $\Delta E / E \sim 0.25^{\circ}$ and $11.25^{\circ}$, respectively. The velocity distribution of the ions was measured once every $6 \mathrm{~s}$, which is the spin period of the Polar spacecraft. The upper cutoff energy of the instrument $(\sim 500 \mathrm{eV})$ limits the measurements of the magnetosheath-like plasma and magnetospheric hot population. Therefore the data from another plasma instrument on Polar, Hot Plasma Analyzer (HYDRA), are combined with the TIDE data to provide identification of the highlatitude boundary layer [Scudder et al., 1995]. The magnetometer (Magnetic Fields Experiment (MFE)) provides the measurements of the magnetic field vector with time sampling of $\sim 0.01 \mathrm{~s}$ [Russell et al., 1995]. The fluxgate magnetometer FM3 on board Interball measures the magnetic field at a rate of $4 \mathrm{~Hz}$ [Klimov et al., 1997]. Plasma characteristics were obtained by ion and electron spectrometers. The CORALL instrument measures ions with energies $0.3-24 \mathrm{keV} / q$ [Yermolaev et al., 1997]. The analyzer has a fanshaped field of view $\left(5^{\circ} \times 120^{\circ}\right)$ divided into five sectors by elevation. The central view directions of the sensors are $42^{\circ}, 66^{\circ}$, $90^{\circ}, 114^{\circ}$, and $138^{\circ}$, respectively. During one spin of the satellite ( $\sim 2 \mathrm{~min}$ ) around its axis, which points to the Sun, $32 \mathrm{E} / \mathrm{q}$ spectra are accumulated, and a three-dimensional (3-D) ion distribution function can be constructed. The plasma spectrometer SKA-1 [Vaisberg et al., 1997] provides fast 3-D measurements of ions. It has two identical units with $16 \mathrm{E} / \mathrm{q}$ narrow-angle analyzers and electrostatic scanners. One of the detectors has a field-of-view axis pointed to the Sun, while the other detector is oriented in the 

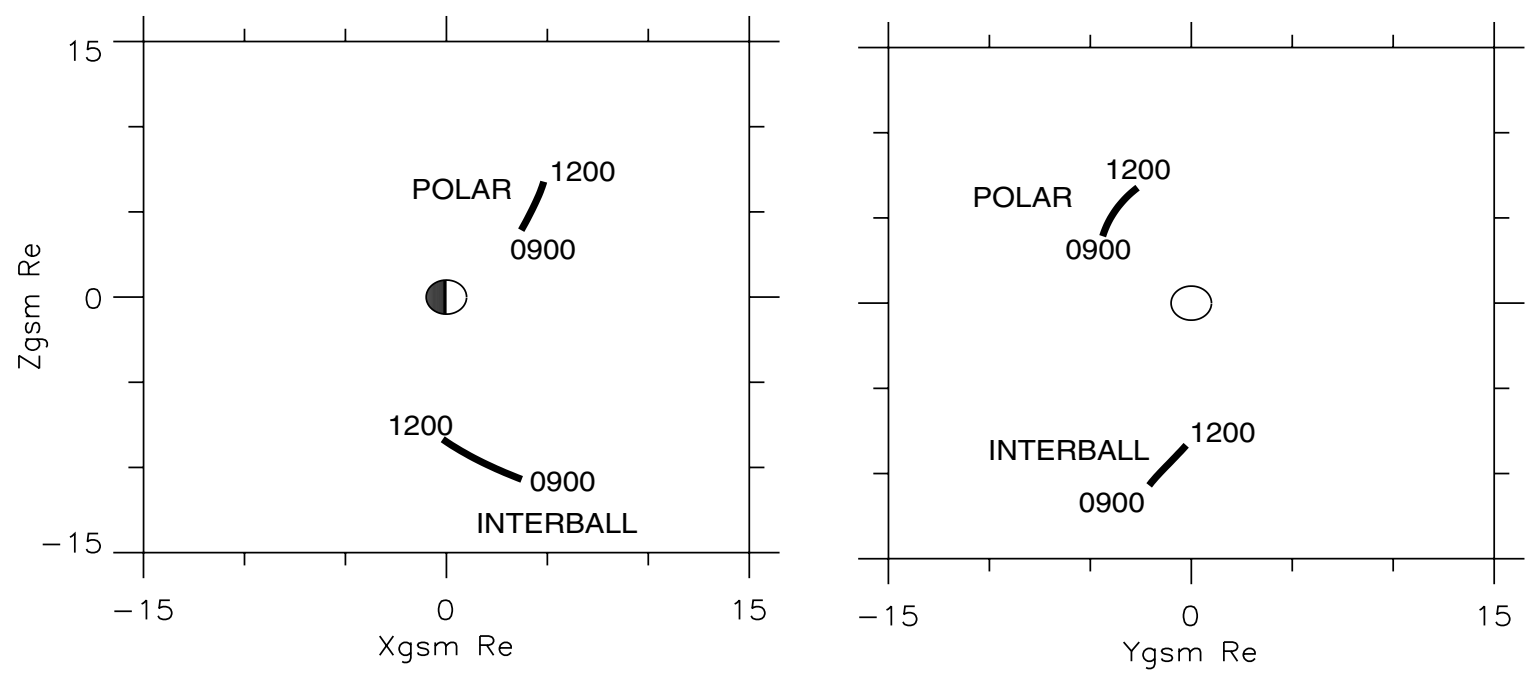

Figure 1. Projections of Polar and Interball orbits onto the $Y Z$ and $X Z$ GSM planes for the time period 0900-1200 UT on 19 June 1998.

opposite direction. The energy range of the instrument is from 50 $\mathrm{eV}$ to $5 \mathrm{keV} / q$. The use of an electrostatic scanning system enables 3 -D measurements in 64 directions in $7.5 \mathrm{~s}$. The electron spectrometer ELECTRON is a "top hat" electrostatic analyzer with a field of view of $180^{\circ} \times 6^{\circ}$. It is divided into eight sectors along the elevation [Sauvaud et al., 1997]. The instrument performs 3-D measurements of electrons in the energy range of $10 \mathrm{eV}$ to $26 \mathrm{keV}$. The full $4 \pi$ solid angle is scanned once per spin of the satellite.

[7] The Geotail measurements are used to monitor the upstream conditions.

\section{Observations}

\subsection{Overall Structure}

[8] Figure 1 shows the projections of Polar and Interball orbits onto the $Y Z$ and $X Z$ GSM planes for the time period 0900-1200 UT on 19 June 1998. Polar surveyed the northern summer highlatitude magnetosphere/magnetosheath along the outbound pass, and Interball observed in the vicinity of the southern winter cusp in the inbound leg. Both satellites were approximately in the same prenoon sector and, perhaps, in magnetically conjugated locations. Figure 2 presents an overall view of the plasma measurements made by each satellite. The top panel shows the spectrogram of electron fluxes measured by the CORALL analyzer on Interball. Regions of different particle characteristics can be clearly identified. At first, the satellite was in the solar wind where particle energy is low and the flux is relatively high. Interball encountered the magnetosheath plasma several times and finally moved into the magnetosheath at $\sim 0730$ UT, identified by an increase in both energy and flux. At $\sim 1000$ UT it went into the region marked by an increase in energy and the appearance of structured electron fluxes, while the electrons at lowest energies disappeared. At $\sim 1130$ UT the intensity of the electron fluxes significantly decreased as the satellite moved into the polar magnetosphere. In contrast to Interball, Polar was, at first, deeply inside the magnetosphere. The middle and bottom panels of Figure 2 show spectrograms of electron and ion fluxes measured by the HYDRA instrument. At $\sim 0830$ UT Polar moved out of the magnetospheric region filled with trapped energetic ions and electrons and at $\sim 0930$ UT entered the polar cap characterized by the distinct drop of the ion fluxes. Significant enhancements of the electron and ion fluxes are observed at $\sim 1000$ UT. After $\sim 1108$ UT a typical magnetosheath plasma was recorded. Over time interval 1000 1130 UT both satellites simultaneously observed similar particle

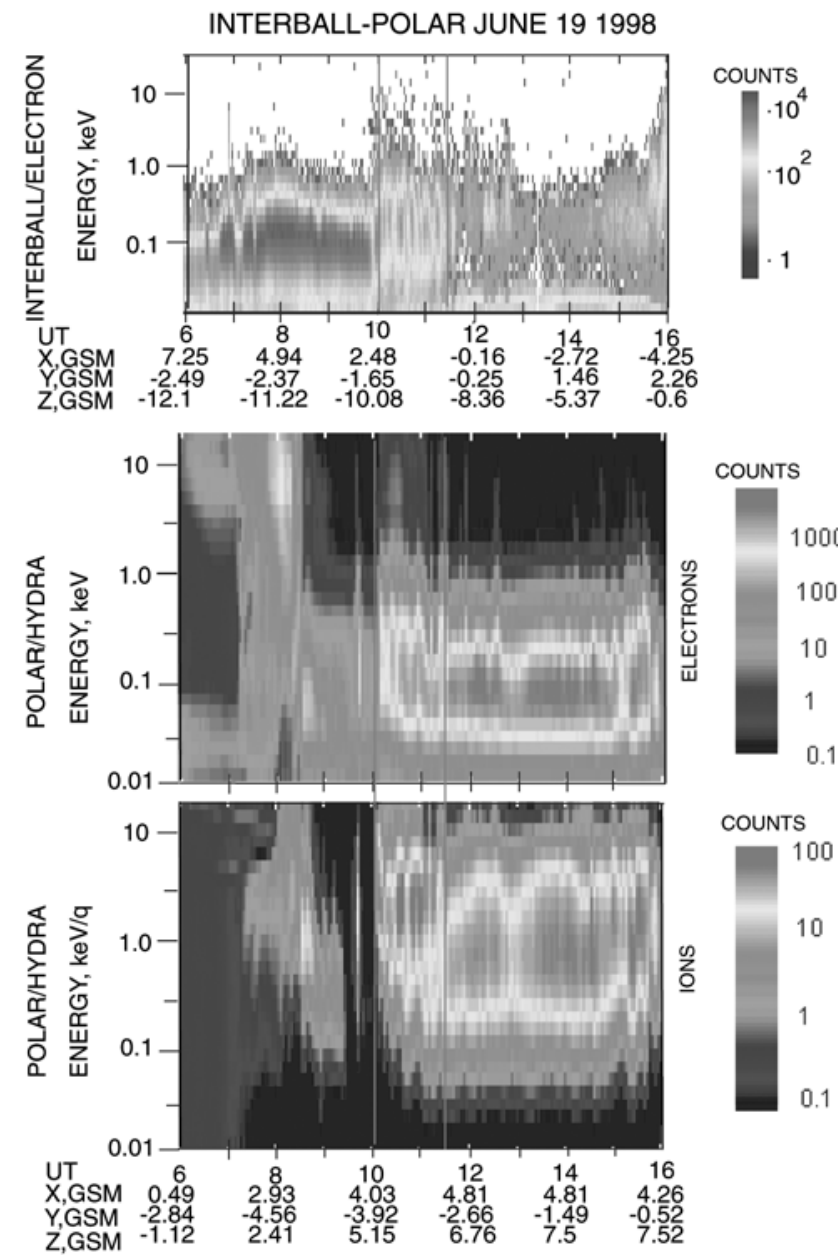

Figure 2. (top) Spectrogram of electron fluxes measured by Interball and spectrograms of (middle) electron fluxes and (bottom) ion fluxes measured by the HYDRA instrument on board Polar are displayed. Red vertical lines bound the time interval when Interball and Polar made coordinated measurements in the southern and northern high-latitude boundary layers close to the cusps. See color version of this figure at back of this issue. 
19 JUNE 1998

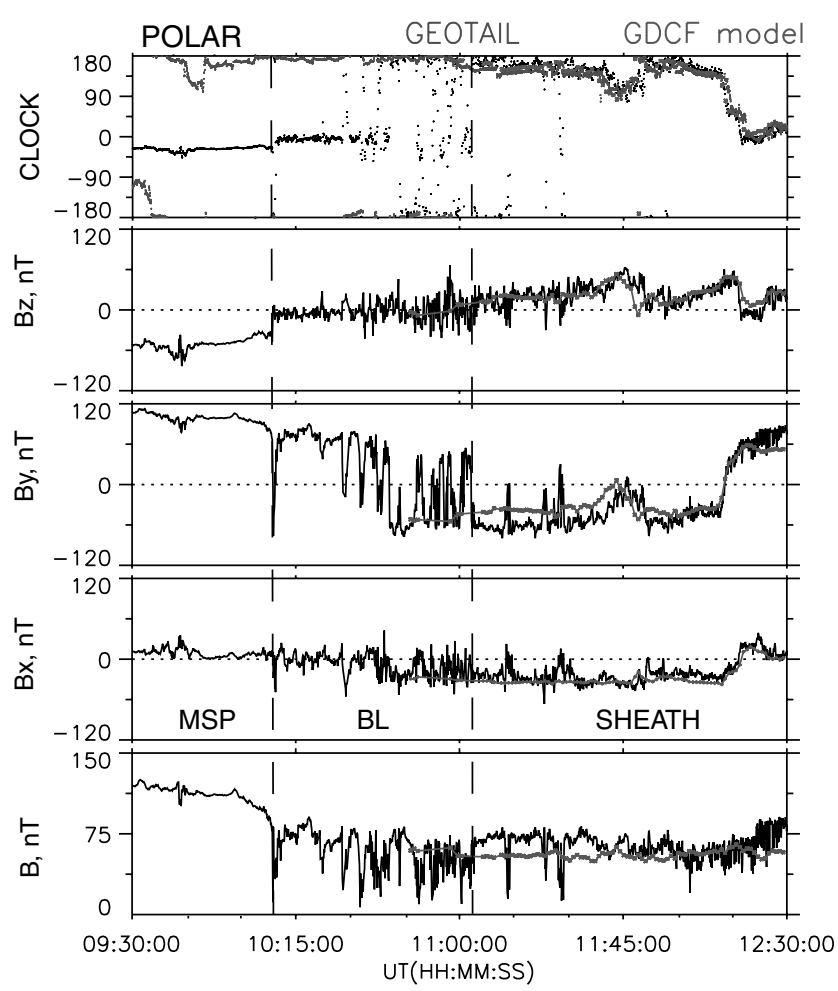

19 JUNE 1998

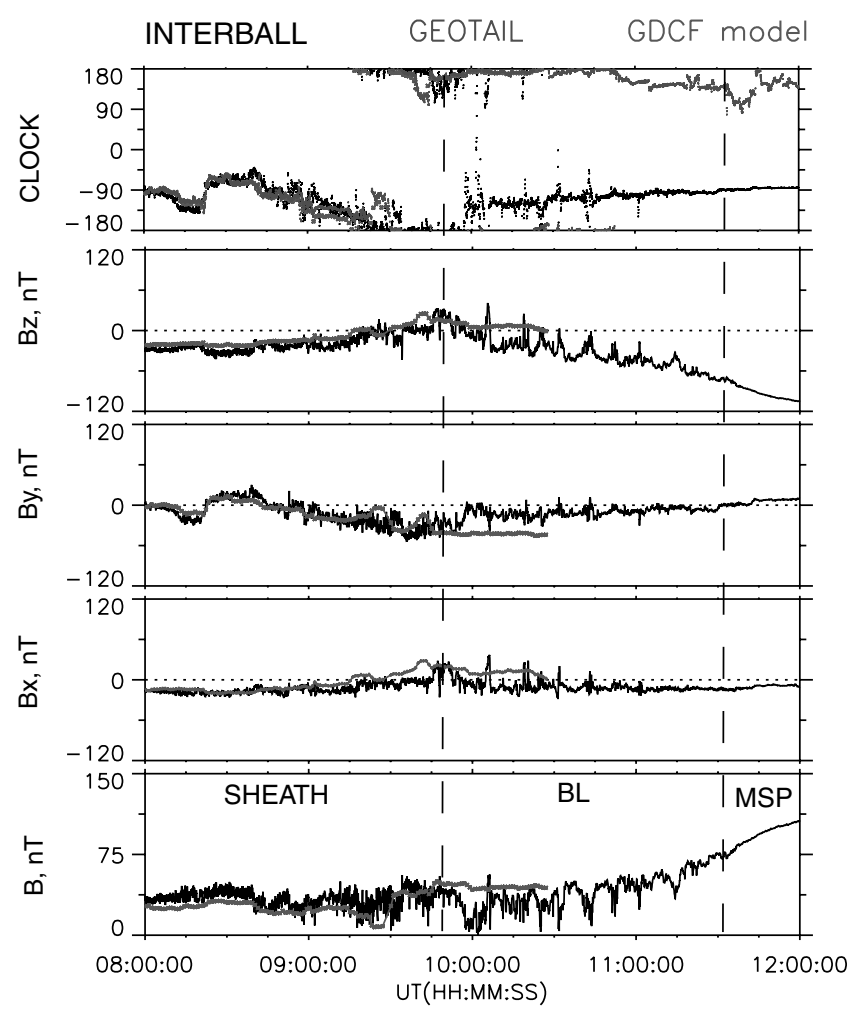

Figure 3. Comparison between the predictions from the gas dynamic model based on the plasma and the magnetic field measurements in the solar wind made by Geotail (red lines) with in situ measurements made by Polar and Interball (black lines). From top to bottom are given the clock angle, three components of the magnetic field in the GSE coordinates, and the magnetic field strength. The clock angle in the IMF with time shift of 10 min to account for the convection time from Geotail to Polar/Interball is plotted by a raspberry line. See color version of this figure at back of this issue.

characteristics. We refer to this region as the distant cusps. Polar surveyed the northern polar cusp while Interball performed the measurements in the region of the southern cusp.

[9] Figure 3 compares the prediction from the GDCF model based on the plasma and the magnetic field in the solar wind measured by Geotail (red lines) with in situ measurements made by Polar and Interball (black lines). From top to bottom are the clock angle, three components of the magnetic field in the GSE coordinates, and the magnetic field strength. The clock angle of the IMF with time shift of 10 min to account for the convection time from Geotail to Polar/Interball is plotted as a raspberry line. There are three adjustable parameters in the GDCF model, namely, a time shift added to the simple convection time, a scale factor adjusting the standoff distance of the magnetopause, and a temperature factor which affects the magnetosheath thickness [Song et al., 1999a, 1999b]. For the Polar case the three adjustable parameters are 6.5 min, 0.8 , and 1 . The time shift of $6.5 \mathrm{~min}$ is added for better fitting of the magnetic field variations, at 1215 UT, for example. The standoff distance is assumed to be $20 \%$ closer to the Earth than that predicted by the dipole field model. This adjustment also includes the difference in the magnetopause shape at high latitudes which can significantly differ from that in the ecliptic plane because of the indentation near the polar cusps. The gas dynamic model assumes that the magnetopause is axisymmetric with respect to the SunEarth line. For detailed discussion about the application and justification of the GDCF model, the reader is referred to Song et al. [1999a, 1999b]. The magnetosheath can be identified as the region with a good correlation between the IMF clock angle and the clock angle measured by Polar. The good correlation exists because the clock angle does not change significantly across the bow shock, as was pointed out by Song et al. [1992]. The direction of the field predicted by the GDCF model agrees well with the observations, although the prediction of the model field strength is not as good. The GDCF model provides the reference direction for the magnetosheath field and partially the magnetopause location. If the observed field direction is distinctly different from the GDCF model prediction, the satellite was most likely not in the magnetosheath at the time. The magnetosphere proper is clearly identified by Polar in the time interval 0930-1005 UT during which the magnetic field contains little large-amplitude fluctuation. A sudden drop of the magnetic field value at $\sim 1008$ UT could be defined as the current layer at the magnetopause. The region $(\sim 1008-1108$ UT) adjacent to the magnetosheath is characterized by strong variations in the magnetic field strength and direction. Savin et al. [1998] used the term turbulent boundary layer (TBL) to characterize this region just outside of the high-latitude magnetopause. Some of the fluctuations indicate a relationship to magnetospheric field lines in this layer. For example, the dominant $B_{y}$ component has a magnetospheric orientation, although $B_{z}$ is substantially different from the magnetospheric one. The oscillations in $B_{y}$ indicate that the field in this region may have two origins.

[10] The magnetosheath can also be clearly identified in the Interball data. The deviation between the clock angle in the interplanetary space and in the magnetosheath appears at $\sim 1000$ UT. The model prediction is not as good as in the Polar case. The difference between the model field and the observed field becomes significant already at $\sim 0945$ UT. It is worth noting that when Interball approached the magnetopause, the $B_{z}$ component in the magneosheath was mostly southward. As statistical studies show, the subsolar magnetopause location depends not only on the solar 


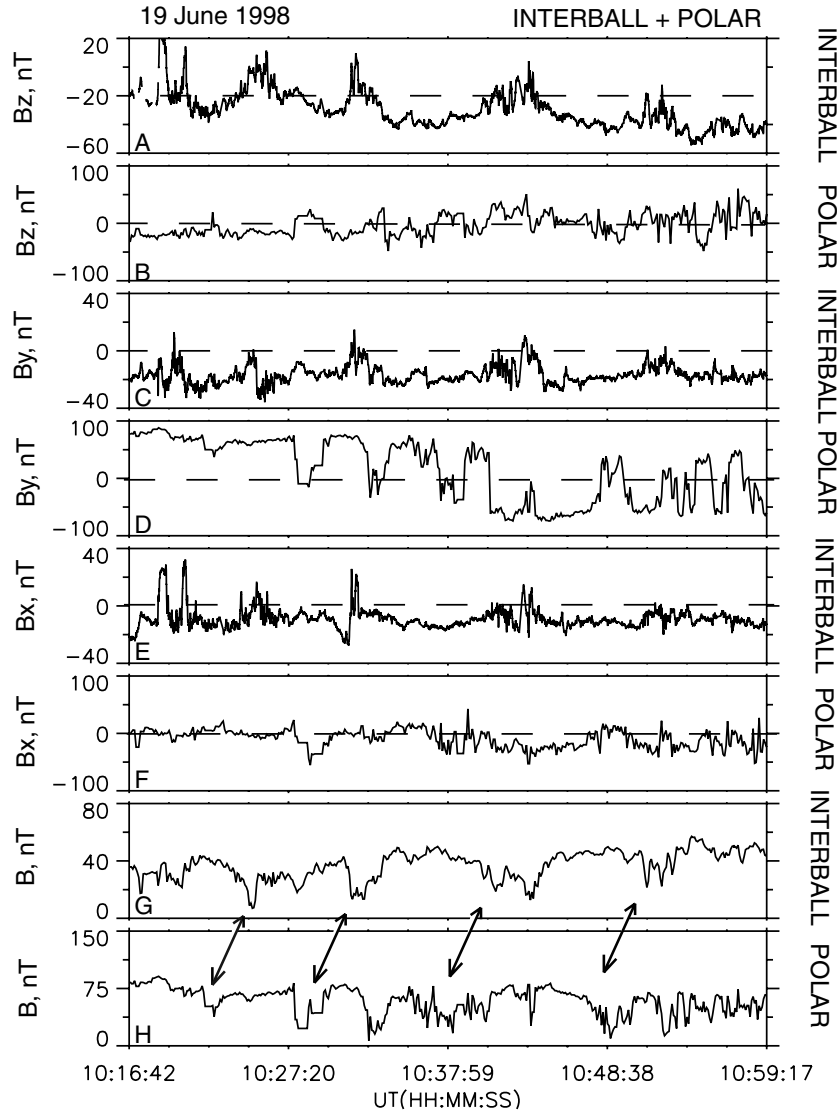

Figure 4. Polar and Interball simultaneous magnetic field measurements for the interval 1016-1059 UT in the northern and southern high-latitude boundary layers, respectively. There are observed similarities.

wind dynamic pressure but also on the IMF $B_{z}$ [Fairfield, 1971; Petrinec et al., 1991; Shue et al., 1997]. The $B_{z}$ dependence of the magnetopause location was not included in the GDCF model. This might be one of the possible reasons for the differences between the model predictions and the observations. At this time interval, Polar was inside the magnetosphere. The "turbulent boundary layer" is clearly observed by Interball at $\sim 1000-1100$ UT. The boundary layer is characterized by the existence of a series of small-scale magnetic cavities within which the magnetic field strength is significantly depressed and the field direction strongly varies. It will be shown later (see Figure 6) that these cavities contain heated/accelerated electrons.

[11] Figure 4 compares the Polar and Interball simultaneous magnetic field measurements for the interval 1016-1059 UT as they crossed the northern and southern high-latitude boundary layers, respectively. Within the boundary layers, nearly quasiperiodical, every $\sim 3-4 \mathrm{~min}$, oscillations were observed by both satellites with a possible small time shift as indicated by the arrows in the bottom panel. There is a possibility that the observed similarities in the structures are due to the conjugate locations of the two satellites. The magnetospheric field is positive (negative) in the $B_{y}$ component at the Polar (Interball) location. The reversals (or strong decreases) of the $B_{y}$ component in the multiple events are accompanied by significant drops in the magnetic field strength.

[12] There are two possible interpretations for the variations in the magnetic field strength and orientation. They can either be multiple magnetopause crossings as a result of radial motions of the boundary due to pressure pulses in the solar wind, or a series of individual structures associated with reconnection events. The conditions in the solar wind were relatively quiet (variations of the solar wind dynamic pressure were $<20 \%$ ) making the boundary oscillations an unlikely interpretation. Observations indicate that the second scenario is more plausible. Figure $5 \mathrm{a}$ presents the angular distribution of ion energy fluxes measured by TIDE in the boundary layer. The direction of the spin-averaged magnetic field is marked by crosses (the earthward direction). The antiparallel direction is indicated by minus signs. It should be noted that the 3-D magnetic field vector had a component outside of the field of view of the TIDE sensors. The energy flux of ions is coded by color. Figure $5 \mathrm{~b}$ gives the energy-time spectrogram of ion fluxes. Figures $5 \mathrm{c}$ and $5 \mathrm{~d}$ show the magnetic field variations. There are several interesting features. First, the transients characterized by a reversal of the $B_{y}$ component and a significant depression in the field strength are accompanied by plasma bursts above $100 \mathrm{eV}$. These ion bursts give rise to a striped structure of the boundary layer clearly seen on the ion spectrogram measured by the HYDRA instrument (Figure 2). Second, these events bound the regions with distinct field-aligned ion fluxes. Although the magnetic field strength and its direction are restored after the passages of transient structures, the plasma characteristics are drastically changed by appearance of ion fluxes traveling toward the Earth along the magnetic field. Acceleration of ions observed in these regions is another important feature.
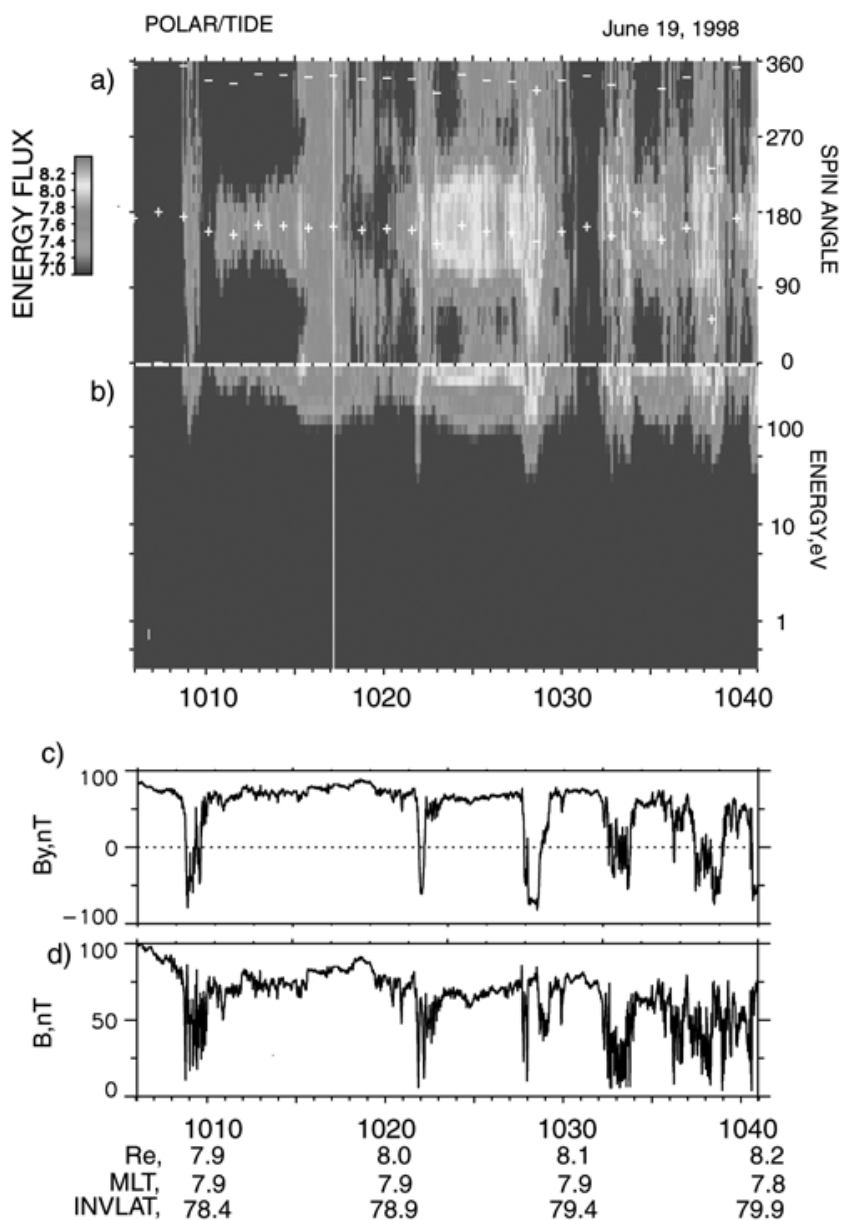

Figure 5. (a) Angular distribution of ion energy fluxes measured by TIDE in the boundary layer. The direction of the spin-averaged magnetic field is marked by pluses. The antiparallel direction is indicated by minus signs. The energy flux of ions is coded by color. (b) Energy-time spectrogram of ion fluxes. (c and d) Variations in the $B_{y}$ component and the magnetic field strength, given for reference. See color version of this figure at back of this issue. 

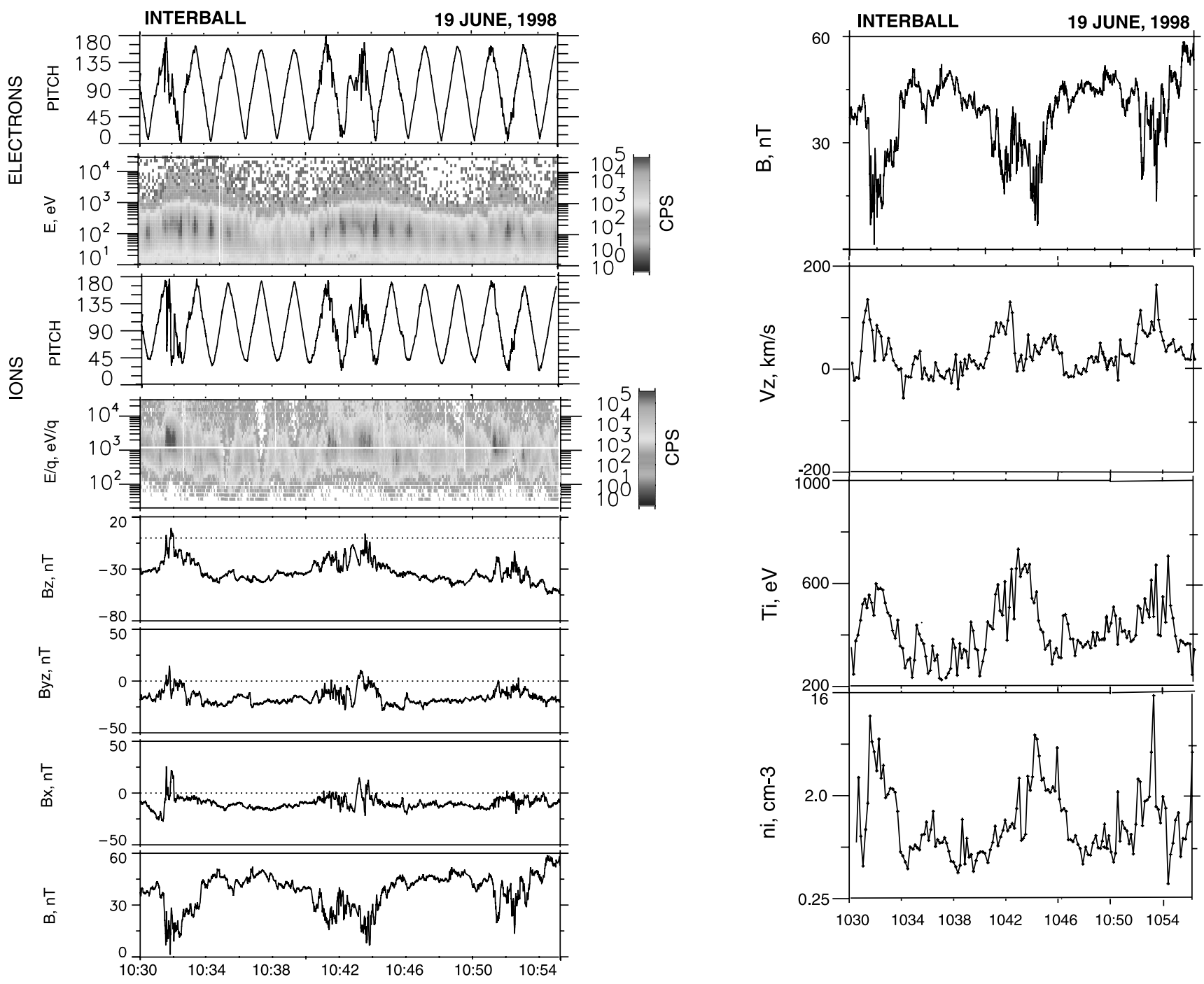

Figure 6. The fine structure of the events observed by Interball is shown in the particle and field measurements. On the left, energy-time spectrograms of ions and electrons, pitch angles of particles, and the magnetic field variations are given. On the right the plasma moments, namely, the component $V_{z}$ (GSE) of the bulk speed, the ion temperature, and the density, are displayed. The top right panel shows the magnetic field strength for reference. See color version of this figure at back of this issue.

Although the TIDE instrument measures only the low-energy part of ion distribution, the effect of ion acceleration is evident. Ions with higher speeds are observed just at the field reversal sites, and most fluxes are beyond the TIDE energy coverage. Third, there is a gradual decrease in the minimum energy of ions streaming along the magnetospheric field. It is clearly seen, for example, during the period 1010-1018 UT following the event at 1009 UT. These signatures are typical of reconnection.

[13] Similar features were also observed by Interball surveying at the same time interval in the conjugate location of the boundary layer. Figure 6 shows the energy-time spectrograms of ions and electrons measured by the ELECTRON and CORALL instruments, respectively, pitch angles of particles, and the magnetic field strength. Bidirectional field-aligned fluxes of the electrons indicate that at least a part of the region is on closed field lines. Observations of trapped energetic ions with nearly $90^{\circ}$ pitch angles support this conclusion. Within the transients, which can be easily identified by strong depression of the magnetic field strength, the mean energy of electrons increases and high-energy tails appear. A bidirectional field-aligned pattern of the electron fluxes is also seen. Ion fluxes also reveal evident features of bulk heating and appearance of energetic particles with energies up to $\sim 30 \mathrm{keV}$ streaming anti- parallel to the magnetic field in the earthward direction. It is also seen that the topology of the magnetic field tubes changes after a transient passes by the satellite. A distinct loss cone for high-energy particles centered near $\alpha \sim 180^{\circ}(\sim 1037,1039$, and 1049 UT) and total or partial cease of bidirectional electron fluxes (1036-1040 and 1047-1050 UT) are consistent with the notion that one end of the field lines loses its connection with the Earth. It is worth noting a "wedge-shaped" pitch angle distribution of ions with a weak top observed at 1035-1040 UT. Such structures are often recorded by Interball in the region adjacent to the high-latitude magnetopause near the polar cusps [Fedorov et al., 2000]. A wedge-shaped distribution can result from particles that have large pitch angles and move slowly parallel to the magnetic field tailward. An interesting point is that trapped suprathermal ions $(>1 \mathrm{keV})$ with pitch angles $\alpha \sim 90^{\circ}$ take a longer time before they escape from the open field lines. These particles appear to be quasi-trapped in the cusp field geometry [Fedorov et al., 2000].

\subsection{Fine Structure}

3.2.1. Magnetic field features. [14] In this section we will analyze the structure of these events in more detail. One of the 
POLAR/10:32:59-10:34:37

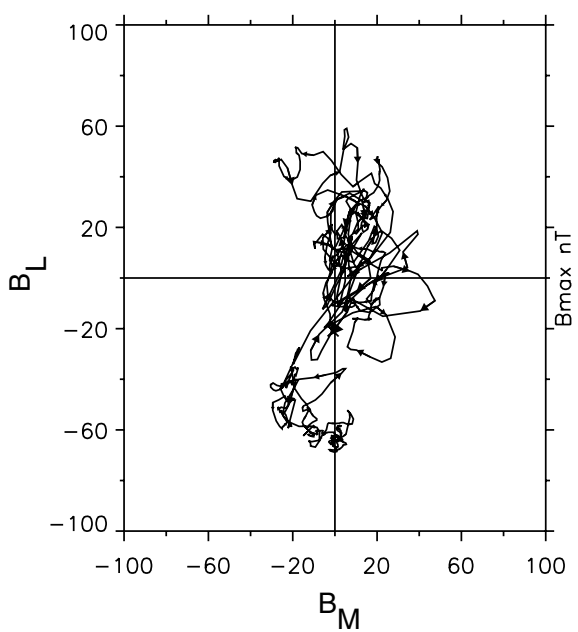

INTERBALL/10:18:04-10:19:06

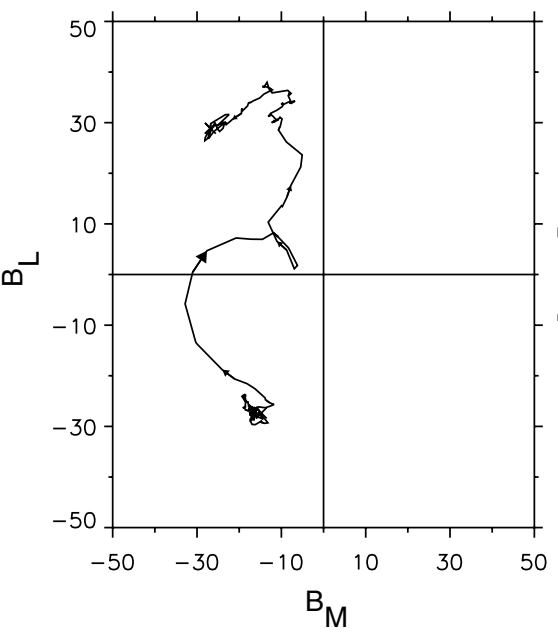

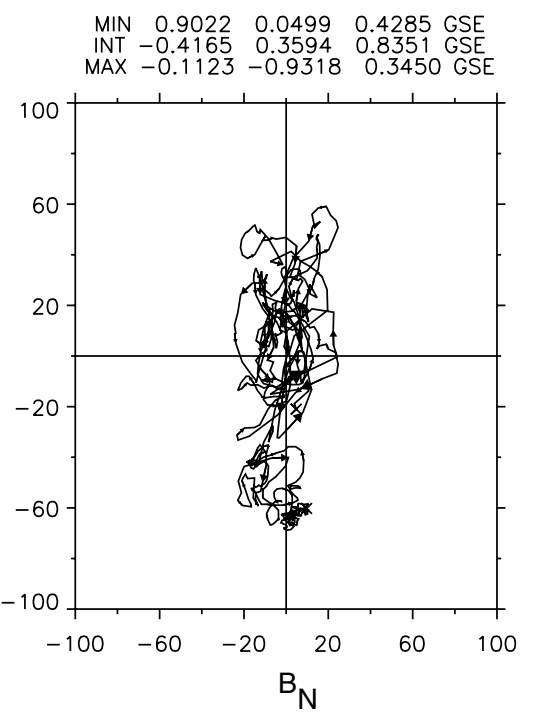

$\begin{array}{llll}\text { MIN } & 0.7529 & 0.3188 & -0.5758 \text { GSE }\end{array}$

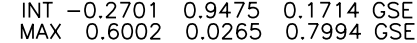

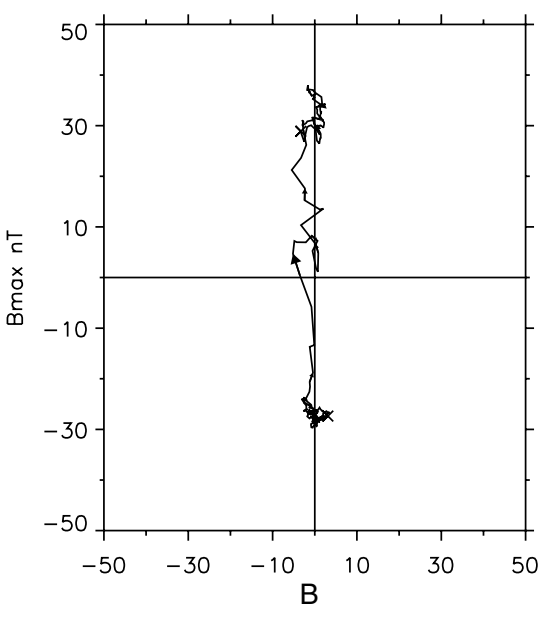

Figure 7. Examples of S-shaped field rotations observed by Polar and Interball. The data are shown in the principal axes determined from the minimum variance analysis. Eigenvectors min $(N)$, Int $(M)$, and max $(L)$ are given in GSE coordinates.

features of the transient events is the S-shaped polarization of the magnetic field variations. Figure 7 gives examples of the S-shaped rotations recorded by both Polar and Interball. In the first case, Sshaped rotation is revealed on the background of smaller-scale field variations. The S-shaped rotation of the magnetic field is a typical signature for rotational discontinuities or intermediate shocks. A striking feature of most of the transients is an obvious asymmetry with a steep ramp followed by trailing oscillations, or vise versa with a wave train upstream of a steep front. Figure 8 shows smallscale variations in the magnetic field strength for several events observed by Polar and Interball.

3.2.2. Plasma features. [15] A similar asymmetry is also seen in the behavior of the fluid parameters of the plasma, number density, velocity, and temperature. The right panels in Figure 6 show variations in the ion velocity, temperature, and density measured by Interball SKA-1 when the satellite was on the magnetospheric field lines. The magnetic field strength is given for reference. The events characterized by strong depression of the strength in the magnetic field are accompanied by "injection" of magnetosheath-like plasma. Ion measurements made by Interball clearly show that the velocity of ion jets exceeds the bulk velocity in the magnetosheath, indicating that plasma is accelerated. Steep fronts with leading or trailing magnetic field oscillations have steep fronts and wave counterparts in the variations of the plasma parameters.

[16] Flow patterns and kinetic characteristics of ion distribution functions measured by Polar also reveal signatures of transient reconnection. To compare behavior of the magnetic field and plasma flow, we plot 2-D vector diagrams of the ion velocity and the magnetic field in the orbital satellite coordinates. The $X_{\mathrm{S} / \mathrm{C}}$ and $Y_{\mathrm{S} / \mathrm{C}}$ axes are in the spin plane, and the $Z_{\mathrm{S} / \mathrm{C}}$ axis coincides with the spin axis of the satellite, where $\mathrm{S} / \mathrm{C}$ stands for spacecraft. It must be noted that after a failure of the mass sensor, TIDE measured only $2-\mathrm{D}$ velocities in the $\mathrm{S} / \mathrm{C}$ spin plane. In our case, the $X_{\mathrm{S} / \mathrm{C}}$ and $Y_{\mathrm{S} / \mathrm{C}}$ axes approximately point to the sunwarddawnward and northward-duskward directions, respectively. The spin axis vector is in the direction $26.3^{\circ}$ declination (GSE) and $202^{\circ}$ right ascension (GSE). The angle between the spin plane and the local magnetic field was $\sim 45^{\circ}$, which means that for the nominal parallel (antiparallel) direction, TIDE is simultaneously 

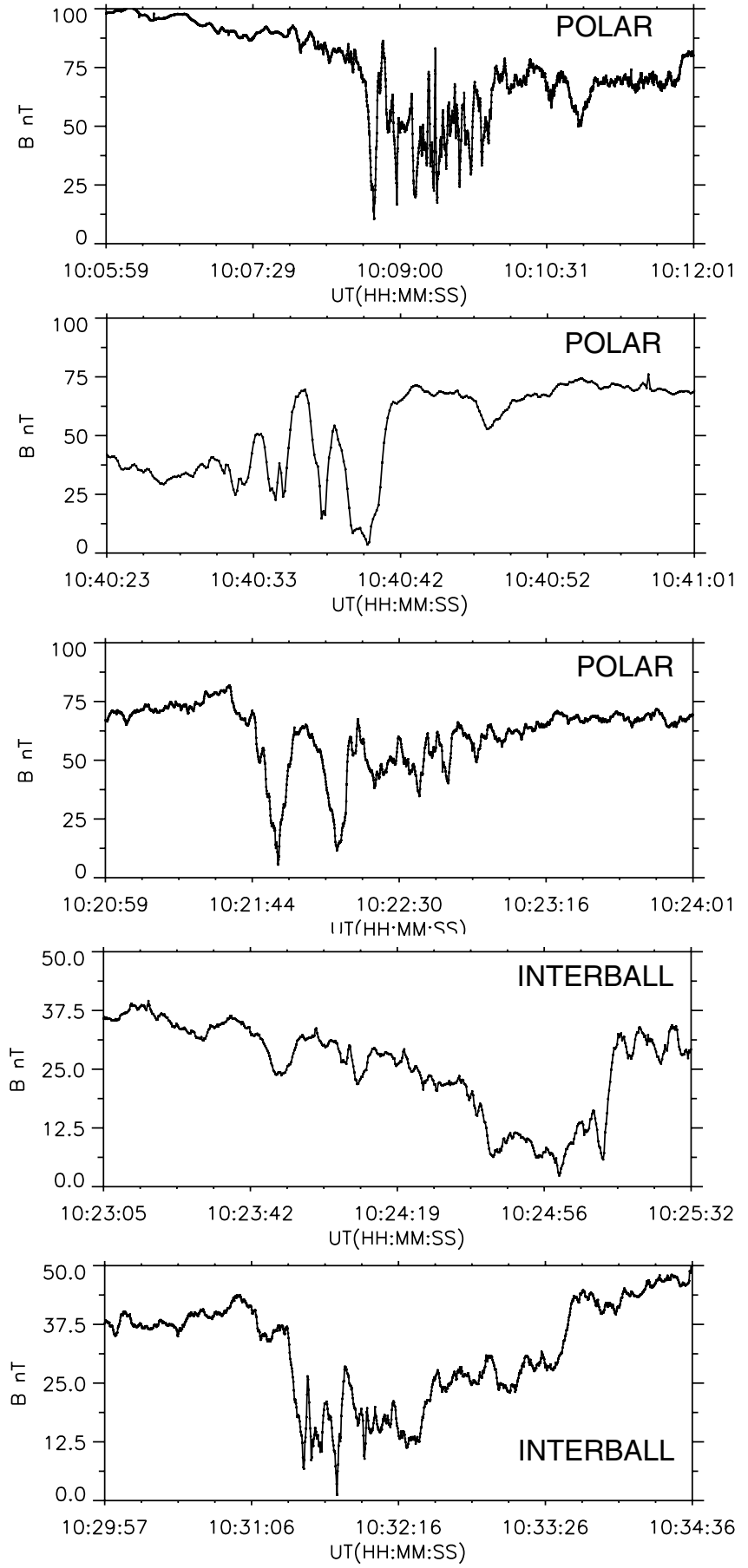

Figure 8. Structure of the events commonly recorded by Polar and Interball is shown here. It is characterized by an obvious asymmetry with a steep ramp followed by trailing oscillations, or vise versa with a wave train followed by a steep front.

sampling a limited range of pitch angles from $0^{\circ}$ to $125^{\circ}\left(77^{\circ}-\right.$ $\left.180^{\circ}\right)$. Figure 9 shows the $V_{1}-V_{2}$ and $B_{1}-B_{2}$ vectors for the time periods 1008-1012 UT and 1014-1019 UT, where subscripts 1 and 2 denote $X_{\mathrm{S} / \mathrm{C}}$ and $Y_{\mathrm{S} / \mathrm{C}}$, respectively. A sketch shown in the bottom right gives the hypothetical trajectory of the satellite in the reference frame of a moving bulge on the magnetopause for one possible scenario. It is based on the field and velocity perturbations and the overall constraint of transient/bursty reconnection. The cartoon at the bottom left illustrates the main elements of a bulge launched by transient reconnection [Southwood et al., 1988; Scholer, 1988; Semenov et al., 1992]. The entry into the bulge occurs with a sharp reversal of the magnetic field at $\sim 1009$ UT and the appearance of plasma flowing as in the adjacent magnetosheath $\left(V_{1}<0, V_{2}>0\right)$. The gradual rotation of the magnetic field at 1009:30 UT is interpreted as a crossing of the region just behind the bulge propagating on the magnetospheric side of the current sheet. Plasma flow also turns around. A part of the flow is out of the bulge. A vortex-like plasma outflow is followed by the observation of quasi-steady ion fluxes streaming parallel to the magnetic field toward the Earth.

[17] It is also useful to look at 2-D ion distributions in velocity space because the moments of distribution functions do not always adequately describe ion properties. It is worth noting that the field-aligned projection of 2-D measurements (shown here) may be different from a full 3-D measurement. Field-aligned fluxes with a D-shaped distribution are seen until $\sim 1018$ UT. The upper row in Figure 10 shows a set of 2-D ion distributions in $V_{\|}-V_{\perp}$ coordinates, where the component $V_{\|}$is the velocity vector measured in the spin plane along the projection of the magnetic field onto this plane. In this coordinate system the component $V_{\mathrm{z}}$ is along the satellite spin axis and the component $V_{\perp}$ completes a right-handed system. Distribution 1 in Figure 10 with ions streaming toward the Earth $\left(V_{\|}>0\right)$ is a typical example of truncated D-shaped distribution. Depletion of ions seen at $V_{\|}<0$ indicates that the other end of the field line is open to the magnetosheath. An interesting, common feature of distributions measured on reconnected field lines (see, for example, distributions 2 and 3 ) is the presence of ions with $V_{\|}<0$ streaming antiparallel to the magnetic field but with lower flux than that parallel to the magnetic field. Such fluxes could be associated with ions reflected somewhere at lower magnetospheric altitudes. The sequence of distribution functions 4-6 measured later shows a gradual increase in the population of the reflected ions. As a result, the distribution functions become more and more isotropic corresponding to the appearance of a stagnant like plasma on the magnetospheric field lines. Indeed, the ion bulk velocities at 1016-1018 UT become very low (Figure 9).

[18] The second row in Figure 10 gives other examples of distributions with counter streaming ions. In distributions 7-8, sequentially measured inside the "magnetic cavity" centered at 1033 UT (Figure 5), we see several narrow ion beams streaming almost antiparallel to the magnetic field. One might argue that these beams are due to temporal effects in the measurements. The similarity of three sequential measurement scans does not seem to support this argument. On the contrary, it is more likely the beams existed throughout the interval and the temporal effects only modulated their magnitude.

[19] Figure 11 shows the plasma flow and the magnetic field in the spin plane $X_{\mathrm{S} / \mathrm{C}^{-}}-Y_{\mathrm{S} / \mathrm{C}}$ during a crossing of the boundary between the inner and outer parts of the boundary layer ( 1040 UT). A "bulge" on the magnetopause from the magnetosheath side can be recognized from the rotation of the magnetic field vector at 1042:30-1043:30 UT. The sequence of events can also be better described in terms of propagating bulges (the sketch at the bottom of Figure 11). Injection of the magnetosheath ions into the bulge is seen from the rotation of the velocity vector. At 1043:45 UT the satellite crossed the reconnected magnetospheric field tube which contained a D-shaped plasma distribution in the $V_{\|}>0$ hemisphere (distribution 10 in Figure 10) and exited the bulge into the sheath. An interesting feature is a small flow vortex at the magnetosheath side of the leading front of the bulge centered at 1042:15 UT. In this vortex, adjacent to the bulge, the plasma flows out of it. Existence of flow vortices is typical of the outer part of the boundary layer located outside the magnetopause. A similar flow pattern with a vortex of plasma flow attached to a bulge was 

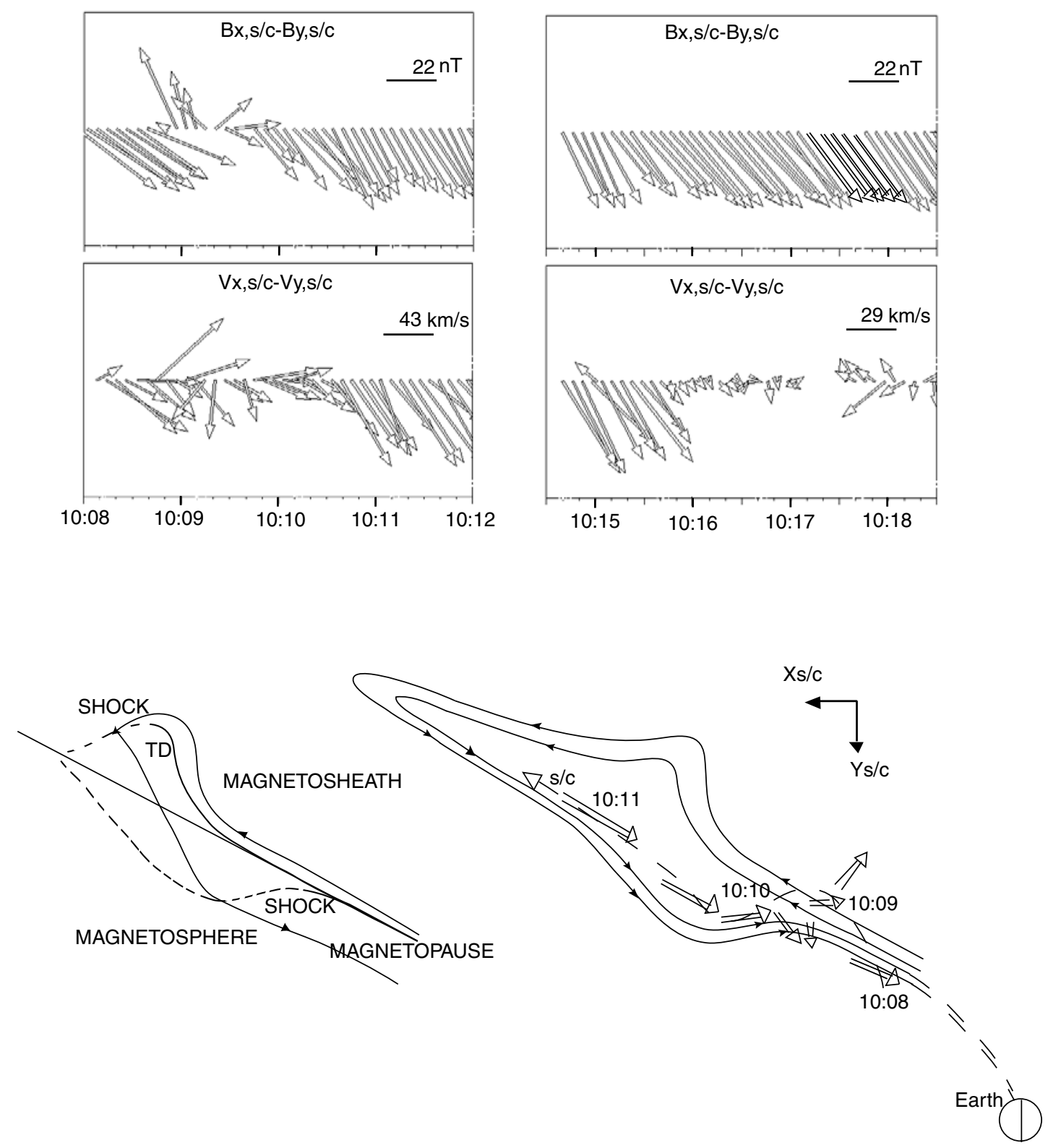

Figure 9. Two-dimensional vector diagrams of the ion velocity and the magnetic field in the satellite coordinates. On the bottom right is a sketch of the hypothetical trajectory of the satellite in the reference frame of a moving bulge on the magnetopause. On the bottom left is a schematic illustrating the structure of a bulge launched by transient reconnection. Interconnection between the magnetospheric and sheath field lines occurs through the shocks. The core region between the magnetosheath and magnetospheric shocks is a field reversal region which contains field-aligned plasma jets [Semenov et al., 1992].

observed in simulations of time-dependent reconnection at the magnetopause [Scholer, 1989].

\section{Discussion}

[20] The coordinated measurements carried out by the Polar and Interball satellites in the northern and southern high-latitude boundary layer (magnetopause-cusp-sheath interface) show that this region is characterized by strong variations of the magnetic field strength and direction. Savin et al. [1998] introduced the term "turbulent boundary layer" (TBL) to emphasize this prominent feature. They assumed that the TBL is located just outside the magnetopause and represents a characteristic feature of the highlatitude magnetopause. Analyzing the structure of this layer by using the magnetic field and plasma instruments on board Polar and Interball, we show that the "turbulence" often consists of recurrent structures characterized by common features. Characteristics of ions and electrons strongly support the idea that the field lines threading the plasma are connected, at least at one end, with the Earth. In other words, the boundary layer is located, at least partly, on reconnected field lines.

\subsection{Source}

[21] Reconnection determines not only the topology of the boundary layer but also the origin and characteristics of the plasma. We associate the observed phenomena with transient reconnection. The events are characterized by a sudden reversal of the magnetic field from the magnetospheric (sheath) to the 


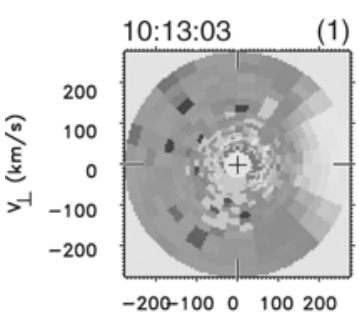

$V_{11}(\mathrm{~km} / \mathrm{s})$

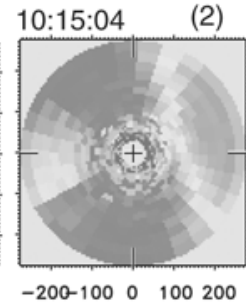

$v_{11}(\mathrm{~km} / \mathrm{s})$

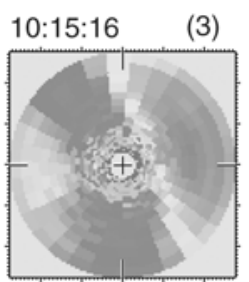

$-200-1000100200$

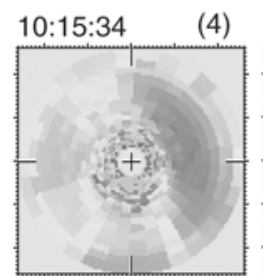

$-200-1000100200$

$V_{11}(\mathrm{~km} / \mathrm{s})$

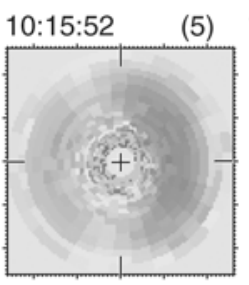

$-200-1000100200$ $V_{11}(\mathrm{~km} / \mathrm{s})$

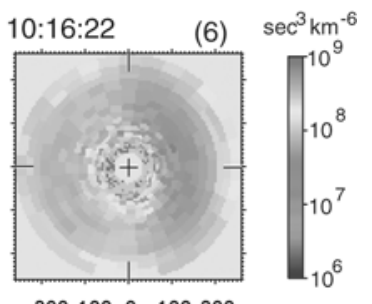

-200-100 $0 \quad 100200$

$V_{11}(\mathrm{~km} / \mathrm{s})$

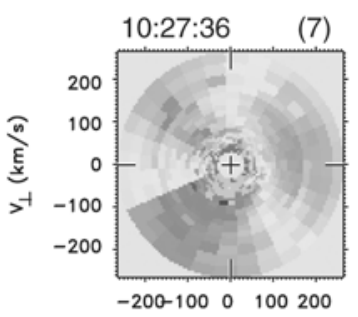

$V_{11}(\mathrm{~km} / \mathrm{s})$

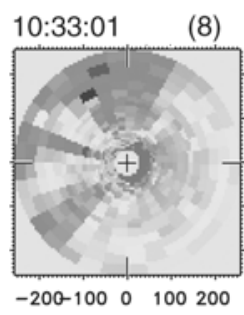

$V_{11}(\mathrm{~km} / \mathrm{s})$

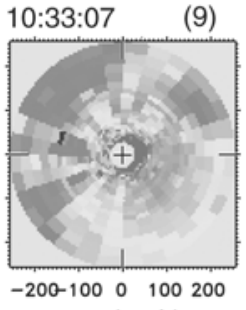

$V_{11}(\mathrm{~km} / \mathrm{s})$

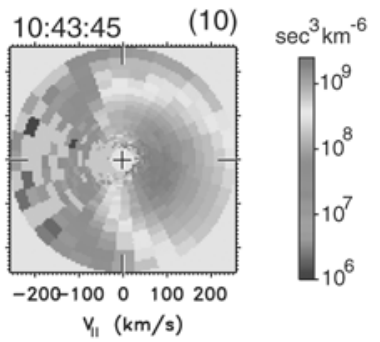

Figure 10. A set of 2-D velocity distributions of ions in $V_{\|-} V_{\perp}$ coordinates. See color version of this figure at back of this issue.

sheath (magnetospheric) direction and by significant depression of the magnetic field strength. Similar events take place repeatedly. Such transients may appear as surface waves on the magnetopause. Observations of surface waves (ripples) are a common feature of the magnetopause. Different sources have been suggested to explain surface waves, including the KelvinHelmholtz instability and pressure pulses in the solar wind [Miura, 1987; Sibeck, 1992]. The events analyzed in our paper may represent a certain class of surface waves. However, plasma characteristics observed in the transients (ion and electron heating, bulk acceleration, and field-aligned fluxes of energetic particles) strongly indicate that these waves are only the manifestation of a more fundamental process operating near the high-latitude magnetopause. It is believed that this fundamental process is time-dependent reconnection between the interplanetary magnetic field and the Earth's magnetic field.

\subsection{Reconnection Location}

[22] Injection of the magnetosheath ions characterized by a steep increase of the ion energy followed by a descending tail in the spectrogram is one of the typical signatures of reconnection. Indeed, ions of different field-aligned velocity injected into the magnetosphere are spatially and temporary dispersed as they arrive at the satellite. An observer near the magnetopause will see reconnected field lines passing by the observation point. Because the satellite velocity is much smaller than the propagation speed of open field lines, the observer will subsequently record the field lines reconnected at different times. Time of flight between the injection and the observation points is $d_{\text {inj }} / V_{i}$, where $d_{\text {inj }}$ is the fieldaligned distance between the injection point where the ion is accelerated and the satellite. Information about the location of the reconnection site can be obtained by measuring the minimum field-aligned energy of the injected ion population [Lockwood and Smith, 1994; Smith and Lockwood, 1996]. The distance from the satellite to the reconnection site may be roughly estimated as $D \sim$ $V_{\text {min }} t \sim 1.5-4 R_{E}$. Here $V_{\min } \sim 65 \mathrm{~km} / \mathrm{s}$ is the minimum fieldaligned ion velocity in the region of the reconnected field lines, and $t \sim 2-6 \mathrm{~min}$ is the time estimated from the injection events at 1009,1022 , and 1033 UT. This is consistent with reconnection at high latitudes for the observed dominant dawnward $\left(B_{y}<0\right)$ and northward $\left(B_{z}>0\right)$ IMF. It is worth noting that determination of the low-energy cutoff also depends on the sensitivity and the energy range of the detector. In this respect, TIDE, which was designed for the measurements of low-energetic ions, is very suitable for monitoring $E_{\min }$. Figure 12 accentuates the low-energy spectrogram of field-aligned ions measured when Polar observed the reconnection event at 1012-1017 UT. We use here the differential energy flux, which makes cutoff much harder. It is seen that ions with energies of $20 \mathrm{eV}$ reach the satellite. The value of the lowenergy cutoff in the spectra of injected ions also contains information about characteristics in the vicinity of the reconnection point. Considering a quasi-steady reconnection with field-aligned adiabatic motion of ions, the minimum energy of injected ions is about $m_{i} V_{\mathrm{dHT}}^{2} / 2 \cos ^{2} \phi$, where $m_{i}$ is the ion mass, $V_{\mathrm{dHT}}$ is the deHoffmann-Teller velocity close to the injection point, and $\phi$ is the angle between the magnetopause and the magnetospheric field line. The dHT speed is determined by the vector sum of the bulk speed of the sheath ions and the local Alfven speed. The small value of the lowenergy cutoff observed by the TIDE instrument indicates that if the angle $\phi$ is not close to $90^{\circ}$, the bulk speed and Alfven speed in the region of the $\mathrm{X}$-point are less than their typical values in the adjacent magnetosheath.

\subsection{Walen Relation Test}

[23] Acceleration of the plasma flowing into a field reversal region is the result of stress $\left(\mathbf{j} \times \mathbf{B}_{n}\right)$ forces associated with the normal component of the magnetic field. The tangential stress balance across the rotational discontinuity is often described in the deHoffmann-Teller reference frame (dHT), in which there is no motional electric field $\mathbf{V} \times \mathbf{B}$. In this frame, plasma flows through the discontinuity along the field lines with the local Alfven speed. Transformation back into Earth's frame then gives $\mathbf{V}=\mathbf{V}_{\mathrm{dHT}} \pm$ $\mathbf{b} V_{A}$. This Walen relation predicts a linear relationship between the flow speed and the magnetic field. The plus or minus signs correspond to flow antiparallel or parallel to the normal component of the field. This relationship is often used to test stress balances. Figure 13 shows the $V_{1}-V_{\mathrm{dHT} 1}$ and $V_{2}-V_{\mathrm{dHT} 2}$ components of the plasma flow in the dHT frame versus the $X_{\mathrm{S} / \mathrm{C}}$ and $Y_{\mathrm{S} / \mathrm{C}}$ components of the local Alfven speed $V_{\mathrm{A} 1}\left(V_{\mathrm{A} 2}\right)$ for the time interval 10071020 UT when Polar was mostly at the magnetospheric side of the bulges. The negative (positive) sign of $V_{\mathrm{A} 1}\left(V_{\mathrm{A} 2}\right)$ corresponds to the magnetospheric region $\left(B_{1}<0, B_{2}>0\right)$. Negative (positive) values of the velocity $V_{1}\left(V_{2}\right)$ show the plasma flows parallel to the magnetospheric field lines. The linear relationship deteriorates at 

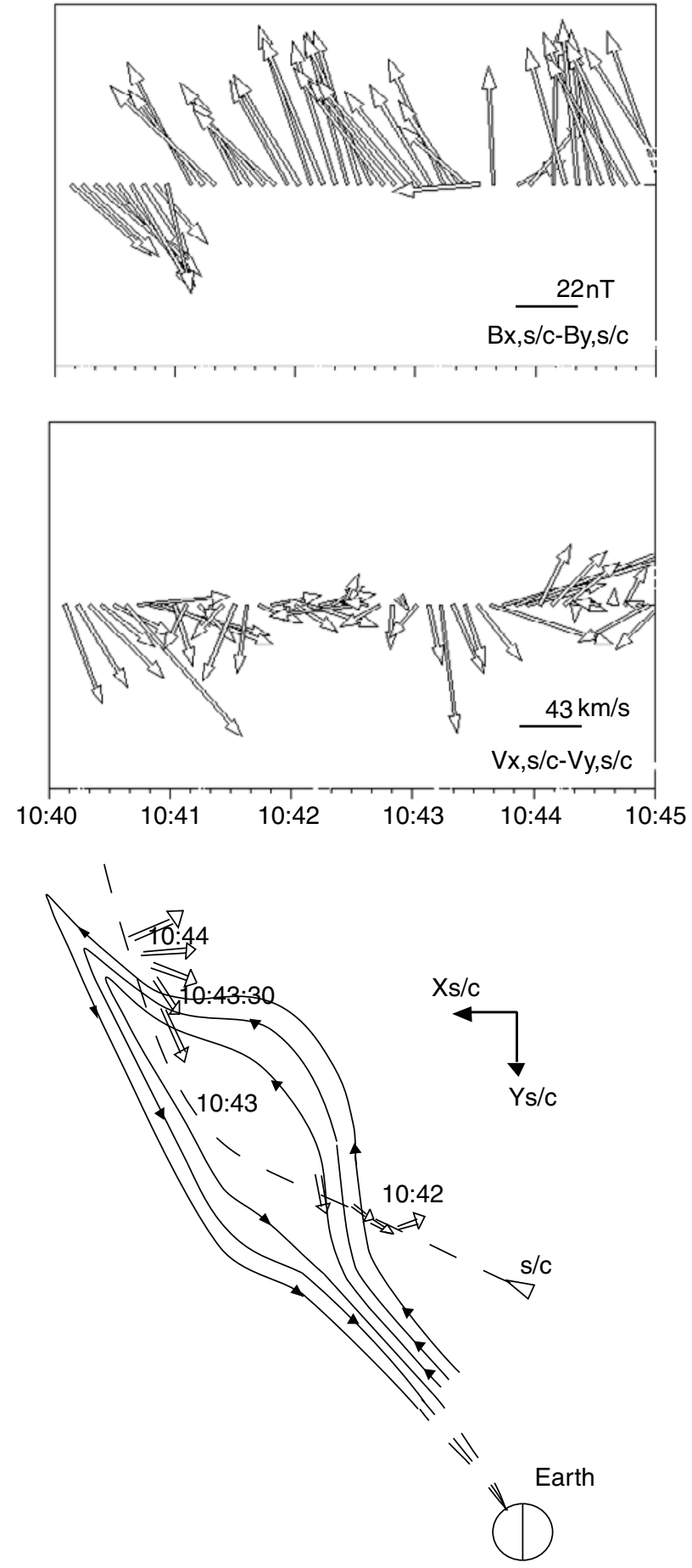

Figure 11. (top) Plasma flow and magnetic field in the spin plane $X_{\mathrm{S} / \mathrm{C}}-Y_{\mathrm{S} / \mathrm{C}}$ near the border between the inner and outer parts of the boundary layer ( 1040 UT). (bottom) Sketch of the hypothetical trajectory of the satellite in the reference frame of the moving bulge.

higher values of the Alfven speed in the inner part of the magnetosphere. The errors in the calculation of the velocity and the density in very rarified plasma may cause a deviation from a linear relation. Although, the agreement between $V_{1,2}$ and $V_{\mathrm{A} 1 \text {, A2 }}$ is not perfect, the positive slope hints at propagation of a kink in the magnetic field (Alfven wave) parallel to the magnetospheric field lines, i.e., in the same direction $\left(-X_{\mathrm{S} / \mathrm{C}},+Y_{\mathrm{S} / \mathrm{C}}\right)$ as plasma jets seen in the numerous bulges. Despite the scatter the trend with a slope less than 1 is clearly seen. The deviation from the ideal Walen slope is not uncommon in Walen relation tests either in interplanetary space, in the magnetosheath, or across the magnetopause. It is worth noting that the HYDRA data also show that in the highlatitude cusp the slope is consistently less than unity [Puhl-Quinn and Scudder, 2000]. Given the errors in the derivation of the plasma moments, this result is not inconsistent with a linear relation between the flow speed and the magnetic field. Systematic analysis of the reasons for a reduction of the Walen slope was made recently by Scudder et al. [1999] and Puhl-Quinn and Scudder [2000]. It is worth noting that a deviation from the Walen relation and a large scatter in the data within the central part of a bulge were also observed in numerical simulations by Scholer [1989]. The scattering increased for time-dependent runs in Scholer's simulations.

\subsection{Ion Kinetics}

[24] Ion distribution functions also contain typical signatures of reconnection. Characteristic D-shaped distributions on the magnetospheric side with cutoff at $\sim V_{\mathrm{dHT}}$ arise because particles experience acceleration by the magnetic field tensions in the same manner as a particle hit by a moving mirror. Additional interesting effects not predicted by simple theories of particle transmission through a rotational discontinuity are also observed. Besides accelerated ions streaming parallel to reconnected magnetospheric field lines, reflected ions with $V_{\|}<0$ appear. Moreover, the population of reflected ions, sometimes, or in some regions, grows and becomes comparable with the population of transmitted magnetosheath ions. As a result, blobs of plasma with "stagnant" properties are observed.

\subsection{Time-Dependent Reconnection}

[25] The data presented in the paper can be best interpreted in terms of transient or pulsating reconnection. A single timedependent reconnection event leads to a pair of bulges at the magnetopause which are transported from the reconnection site approximately with the Alfven speed. In this model, the field reversal regions are bounded by shocks [Scholer, 1988; Semenov et al., 1992]. It is important to note that transient reconnection also acts as an effective mechanism for generating surface waves [Semenov et al., 1992]. The magnetic field and flow perturbations perpendicular to the current sheet propagate with a velocity intermediate between the sheath and the magnetospheric Alfven speeds, giving rise to the appearance of bulges at the magnetopause surface. As a result, on the magnetosheath side, a slow shock occurs at the trailing edge of the bulge, whereas on the
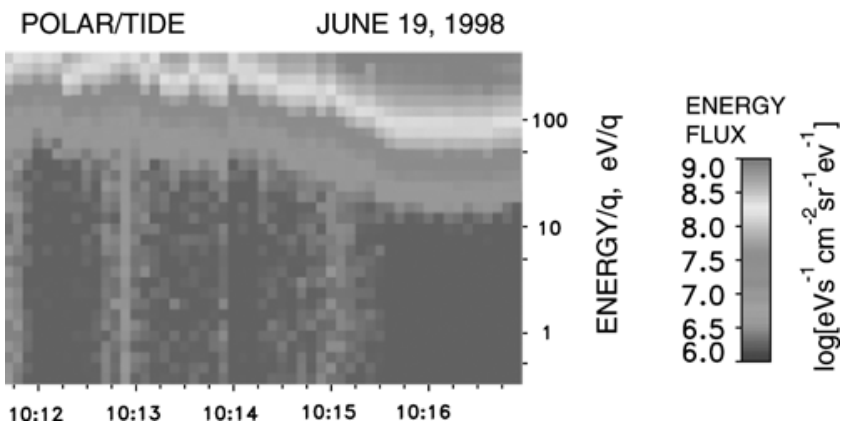

Figure 12. Energy-time spectrogram of field-aligned ions measured when Polar observed the reconnection event at 1012-1017 UT. The value of the low-energy cutoff of injected ions carries information about the distances from the reconnection sites. See color version of this figure at back of this issue. 


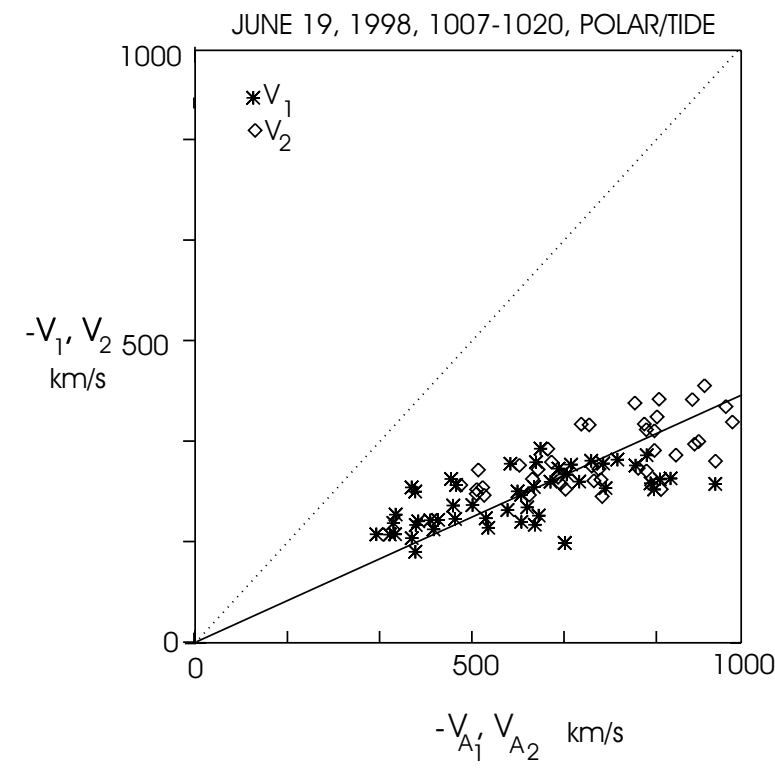

Figure 13. $V_{1}-V_{\mathrm{dHT} 1}$ and $V_{2}-V_{\mathrm{dHT} 2}$ components of the plasma flow in the dHT frame versus the $X$ and $Y$ components of the local Alfven speed $V_{\mathrm{A} 1}\left(V_{\mathrm{A} 2}\right)$ for the time interval 1007-1020 UT when Polar was mostly at the magnetospheric side of the bulges.

magnetospheric side (with higher Alfven speed), a slow shock appears at the leading edge (the sketch at the bottom left of Figure 9). Interconnection between the magnetospheric and sheath field lines occurs through the shocks. The core region between the magnetosheath and magnetospheric shocks is a field reversal region which contains field-aligned plasma jets. Plasma flowing into a bulge through slow shocks is heated and accelerated. Magnetic signatures of a bulge depend not only on the position of the satellite but also on the shock velocities, the direction of their propagation, and other characteristics of the reconnection geometry [Biernat et al., 1998]. In the minimum variance coordinates these signatures often are more complicated than, for example, bipolar variations in the $B_{N}$ component typical for flux transfer events. An entry into or exit from a propagating bulge may occur not only through a shock with rotation of the magnetic field vector but also through a tangential discontinuity [Semenov et al., 1992; Biernat et al., 1998]. Indeed, we see that sometimes exit or entry occurs without a distinct rotation of the field. The flow pattern near bulges contains regular structures. There are signatures of vortex flows into/out of bulges in Figures 9 and 11. The observed picture resembles the flow pattern with flow vortexes attached to a bulge in the simulations of time-dependent reconnection at the magnetopause [Scholer, 1989]. The scenario of multiple transient reconnection can reasonably explain many observed features although it is worth noting that the above-cited timedependent reconnection models are based on homogeneous reconnected fields and do not take into consideration the effects of wave propagation through the cusp regions.

[26] When reconnection is switched on, the magnetopause moves inward. As a result of such motion, the satellite approaches closer to the magnetopause and may enter a bulge propagating along the current sheet. The data presented in this paper show that the time intervals when the satellite observes quasi-steady fluxes of field-aligned plasma jetting along reconnected field lines are preceded by short injection pulses. These injections, characterized by the sudden appearance of accelerated ions with dispersed change in the bulk velocity, a significant decrease of the magnetic field strength, and ion and electron heating, correspond to the entry of the satellite into a bulge launched by the onset or activation of reconnection. The observed characteristic size of a bulge $L \sim V_{A} t \sim$ $3 R_{E}$, where $V_{A}$ is the intermediate Alfven speed and $t \sim 1-2 \mathrm{~min}$ is a typical duration of the injection events. Then, for several minutes, the magnetosphere remains open, and we observe stable fluxes of field-aligned accelerated ions with D-shaped distribution. A cease of reconnection or decrease in the reconnection rate forms a new bulge detached from reconnection site and propagating along the magnetopause. Indeed, the observations often show that periods of quasi-steady reconnection are bounded by passages of two bulges which may correspond to switch-on and switch-off bulges. No more open field lines are added after a cease of reconnection. Activation of reconnection generates a new bulge, and the process is repeated.

[27] An important feature shown in Figure 6 is that the sheath plasma (ions and electrons) is bulk heated inside the observed structures. This is consistent with interpretation of the events as slow/intermediate $(\mathrm{S} / \mathrm{I})$ shocks but not as simply rotational/tangential discontinuities of the current layer at the magnetopause. The distinction between intermediate and slow shocks is difficult because their velocities are close. These shocks may convert the magnetic field energy into particle kinetic and thermal energy. It is worth noting that the spacecraft observations at the low-latitude magnetopause have not yet confirmed the existence of slow shocks although slow shocks play a crucial role in the wave reconnection theories [Petschek, 1964]. On the other hand, Feldman et al. [1985] found that in the distant tail, slow shocks are a semipermanent feature. The data presented in this paper show that the transition through a bulge is associated with an increase in number density, flow velocity, and temperature and a decrease in the magnetic field strength. Because of these properties we referred to the transition as a S/I shock. Although strict identification of S/I shocks needs a test of Rankine-Hugoniot relations, we refer to the observed structures as $\mathrm{S} / \mathrm{I}$ shocks in a more qualitative manner because the observed features have an increase in number density, tangential component of the flow velocity, and temperature and a decrease in the magnetic field strength. A test of Rankine-Hugoniot relations will be performed in a later study. It is worth noting that a S/I shock is predicted by the MHD models but may not necessarily be predicted in a kinetic treatment. However, recent hybrid simulations [Lin and Xie, 1997; Nakamura and Scholer, 2000] have found an intermediate shock which bounds the reconnection layer from the magnetosheath side (the coplanar case). The simulations by Nakamura and Scholer [2000] also exhibit a steep ramp and trailing region (but without periodical wave oscillations). In the noncoplanar case there is almost no difference between an I shock and a rotational discontinuity [Karimabadi et al., 1995; Nakamura and Scholer, 2000].

[28] The fine structure of the transition is rather complicated. S-shaped polarization of the magnetic field variations is a characteristic feature of many bulge crossings. A finite $B_{M}$ arises because of the Hall effects associated with ion inertia. The Sshaped rotation of the magnetic field at the magnetopause was first observed by Berchem and Russell [1982]. Lyu and Kan [1989] found that S-shaped rotation of the magnetic field is a typical signature for Alfven/weak intermediate shocks. Hau and Sonnerup [1990] have shown that such hodograms may occur not only for the weak intermediate shocks but also for the strong ones, wherein the downstream flow is subsonic. S-shaped rotation of the magnetic field was also observed in the kinetic simulations of the intermediate shocks [Karimabadi et al., 1995; Lin and Xie, 1997].

\subsection{Downstream Wave Train}

[29] Another important observation is that the structure of the transition across a bulge often consists of a steep ramp with a wave train (Figure 8). A dispersive wave train in the downstream region is a characteristic feature of weak slow shocks [Coroniti, 1971] in which the magnetic field exhibits helical rotations. It is interesting 
to note that observations in the geomagnetictail did not show experimental evidence of a trailing wave train in reconnection events. Structure of slow shocks was also studied in two-fluid MHD and hybrid simulations. Lee et al. [1989] suggested that a trailing wave train appears only when the upstream Mach number is above a certain critical value. Omidi and Winske [1989] argued that in their 1-D hybrid simulations (low beta regime), these waves exist only when the upstream speed is subsonic and the $T_{e} / T_{i}$ is large enough. More recent studies by Omidi and Winske [1992] have shown that appearance of a wave train is more closely related to the properties of upstream waves generated because of the interaction between the incident and reflected ions. It is important to note that the theories of slow shocks and the simulations predict a rotational wave train with $|B|=$ const whereas in our observations, waves contain a significant compressional component. Generally speaking, a trailing wave train may appear on either the Alfven wave branch (rotational waves) or on the slow branch (compressional modes). In the latter case one can expect variations in $|B|$, although the question of why a wave train does not suffer strong damping in heated downstream plasma remains open. Wave train with essential compression oscillations might also be generated because of nonlinear propagation of the shock through the cusp region.

[30] It has been shown in the paper that a wave train may follow a steep ramp or a ramp may be preceded by trailing oscillations. The observed sequence of the variations in the magnetic field or plasma properties depends on whether the satellite is approaching a shock front from the upstream or downstream side. In the former case, one may expect a steep front with a trailing train. Crossing of the shock and entry into reconnected field lines will be accompanied by the observations of injection events with a steep increase of ion energy followed by a descending dispersed tail. Such injection signatures can also be interpreted in terms of time elapsed after reconnection. At first, the satellite is on "fresh" reconnected field lines, and then, it is on the field lines reconnected at early times. If the satellite crosses the shock from the downstream side, the sequence of observed events will be reversed.

\subsection{Magnetic Field Topology and Region of Stagnant Plasma}

[31] According to our scenario the observed depression of the magnetic field accompanied by plasma acceleration and heating corresponds to crossing of the magnetospheric or magnetosheath slow/intermediate shock bounding a bulge propagating along the magnetopause. The shock is followed by a core region with reconnected magnetic field lines. Field-aligned fluxes of injected magnetosheath ions, the absence of bidirectional electrons or the appearance of asymmetry in their pitch angle distribution, a wedgeshaped pitch-angle ion distribution typical for the lobes, and loss cones in ion fluxes in the earthward direction are observed after the passage of a "switch-on bulge." All these features support the interpretation that the magnetic field lines, which were previously closed, become temporarily open. When reconnection ceases, a switch-off bulge, which propagates with the Alfven speed, is followed again by a tangential discontinuity; that is, the magnetosphere is closing. The events observed at $\sim 1018$ and 1029 UT (Figure 5) may be tentatively interpreted as the passage of switchoff bulges.

[32] One of important questions to be addressed is the topology of the high-latitude boundary layer during time intervals when the reconnection ceases. The observations give a hint that some part of the plasma exposed to reconnection processes remains in this layer after the passage of reconnected field tubes. This gives rise to a stagnant plasma population. The temperature of stagnant plasma is higher than in the adjacent magnetosheath. The stagnant plasma is recurrently supplied with new blobs of hotter and denser plasma carried by freshly reconnected field tubes. Both these populations form the entry layer. Similar reasoning could be applied to "turbulent" plasma in the outer boundary layer adjacent to the magnetopause on the sheath side. One may assume that this layer corresponds to the region of stagnating plasma observed by Paschmann et al. [1976] in the outer cusp. A seemingly disordered plasma flow is managed by flow around multiple large-amplitude ripples propagating along the magnetopause surface and by plasma fluxes flowing in (out) to (from) the magnetosphere. A vortex-like behavior of the flow pattern is the typical signature of this region. Plasma remnants after transits of magnetosheath slow shocks as well as the energetic particles escaping the magnetosphere along there connected field lines contribute plasma population in the outer boundary layer too.

[33] An interesting question is whether the phenomena observed by both satellites are conjugate. The orientation of the IMF $\left(B_{z}>0\right.$, $B_{y}<0$ ) was favorable for macroscale reconnection in the region of the northern polar cusp at the prenoon sector, where the IMF and the magnetospheric fields are antiparallel. The TIDE observations of ion fluxes parallel to the magnetic field during the reconnection events indicate that reconnection sites were at 2-4 $R_{E}$, farther from the Earth along the field with respect to the satellite. At the same time, Interball surveyed the region near the southern cusp and also observed the injection events. The plasma was jetting toward the Earth, antiparallel to the magnetic field lines. The S/I shocks generated during transient reconnection processes move along the magnetopause surface approximately with the local Alfven velocity $\pm V_{A}$ but simultaneously convected with the bulk plasma speed $V_{\text {bulk. }}$. It is difficult to evaluate accurately the time shift between the arrival of shocks at Polar and at Interball. This is due to variations in Alfven and plasma speeds and uncertainty in the location of the reconnection cite. Observations indicate that a value $\Delta t \sim 5-10 \mathrm{~min}$ seems reasonable.

[34] According to the Tsyganenko [1995] (T95) model the satellites were not on conjugate field lines. The T95 model predicts that Polar crossed the region of closed field lines and went into the northern polar cap while Interball remained on the open field lines of the southern polar cap sliding near the separation surface between open and closed field lines. It is worth noting that the T95 model has limitations when applied to the cusp region. The $B_{x}$ and $B_{y}$ components during the magnetospheric intervals measured by Interball have systematic deviation from those of the T95 model. The observations show that the field lines crossed by Interball during the period of coincident observations were closed in the time intervals between the reconnection events. On the other hand, the observations by Polar indicate that the satellite was mostly on the open field lines. Therefore, although the magnetospheric field measured by the two satellites may be conjugate, the cusp signatures may not be conjugate. Despite the fact that similar magnetic field structures were observed by both satellites with a time delay between the satellites of 3-4 min (Figure 4) we prefer to relate these field structures to reconnection at different sites. We believe that the visible similarity of the events at the two satellites is due to the unique structure of single transient reconnection events. The cusp regions are the sites where bulges with $\mathrm{S} / \mathrm{I}$ shocks generated by reconnection processes are focusing because of the cusp field topology. The structure of these events is similar (Figure 8). These elementary shocks, propagating from different sites, and probably interacting with each other in the polar throats, determine a global pattern of the magnetopause-cusp-sheath interface consisting of large-amplitude ripples (bulges) through which the magnetosheath and magnetospheric field lines are connected.

\section{Conclusions}

[35] A number of conclusions can be drawn from this work. (1) The high-latitude boundary layer in the near-cusp region occupies closed and open field lines. Plasma characteristics in the boundary layer differ from that in the adjacent magnetosheath and magneto- 
sphere. An essential part of the boundary layer is on reconnected magnetic field lines. (2) The boundary layer has typical elementary structures which are characterized by a steep ramp followed by trailing oscillations. We assume that these structures are slow/ intermediate shocks bounding surface bulges propagating on the magnetopause generated at transient reconnection events. (3) Population of stagnant plasma also appears in the boundary layer. This occurs because part of ions transmitted into the magnetosphere along there connected field lines are effectively reflected and pitch angle scattered, giving rise to counterstreaming fluxes and quasi-isotropic distributions.

[36] Acknowledgments. The work of E.D. was supported by NATO Collaborative Linkage grant 975277 . The work at UM was supported by NASA under the UM Corporate Agreement NCC8-84 by NASA grant NAG5-1557, by NSF under award NSF-ATM9729775, and by NSF-ONR under award NSF-ATM9713492. The work at UCLA was supported by NASA under research grant NAG5-7721. We would like to acknowledge L. Frank (Geotail CPI) and S. Kokubun (Geotail MGF), whose data obtained on the Web pages were used in our analysis.

\section{References}

Berchem, J. W., and C. T. Russell, Magnetic field rotation through the magnetopause: ISEE-1 and 2 observations, J. Geophys. Res., 87, 8139 1982.

Biernat, H. K., V. S. Semenov, and R. P. Rijnbeek, Time-dependent threedimensional Petschek-type reconnection: A case study for magnetopause conditions, J. Geophys. Res., 103, 4693, 1998.

Chandler, M. O., S. A. Fuselier, M. Lockwood, and T. E. Moore, Evidence of component merging equatorward of the cusp, J. Geophys. Res., 104, 22,623, 1999.

Coroniti, F. V., Laminar wave train structure of collision less magnetic slow shocks, Nucl. Fusion, 11, 261, 1971.

Crooker, N. U., Dayside merging and cusp geometry, J. Geophys. Res., 84, 951, 1979.

Dubinin, E., and I. M. Podgorny, Particle precipitation and radiation belt in laboratory experiments, J. Geophys. Res., 79, 1426, 1974.

Dubinin, E., and Y. Potanin, The phenomena at the magnetospheric boundary and the convection in the polar cap, Cosmic Res., 19, 409, 1981.

Dubinin, E., I. M. Podgorny, and G. G. Managadze, The simulation of penetration of fast particles in the region of neutral points of the magnetosphere, Geomagn. Aeron., 4, 733, 1971.

Dubinin, E., I. M. Podgorny, and Y. Potanin, The experimental evidence of opened and closed magnetosphere, Cosmic Res., 16, 87, 1978.

Fairfield, D. H., Average and unusual locations of the Earth's magnetopause and bow shock, J. Geophys. Res., 76, 6700, 1971.

Fedorov, A., E. Dubinin, P. Song, E. Budnick, P. Larson, and J.-A. Sauvaud, Plasma characteristics of high-latitude cusp for steady southward IMF: Interball observations, J. Geophys. Res., 105, 15,945, 2000.

Feldman, W. C., D. N. Baker, S. J. Bame, J. Birn, J. T. Gosling, E. W. Hones, and S. J. Schwartz, Slow mode shocks: A semipermanent feature of the distant geomagnetic tail, J. Geophys. Res., 90, 233, 1985.

Frank, L. A., Plasma in the Earth's polar magnetosphere, J. Geophys. Res., 76, 5202, 1971

Fuselier, S. A., B. J. Anderson, and T. G. Onsager, Particle signatures of magnetic topology at the magnetopause: AMPTE/CCE observations, J. Geophys. Res., 100, 11,805, 1995.

Gosling, J. T., M. F. Thomsen, S. J. Bame, R. C. Elphic, and C. T. Russell, Observations of reconnection of interplanetary and lobe magnetic field lines at high-latitude magnetopause, J. Geophys. Res., 96, 14,097, 1991.

Haerendel, G., and G. Paschmann, Entry of solar wind plasma into the magnetosphere, in Physics of the Hot Plasma in the Magnetosphere, edited by B. Hultqvist and L. Stenflo, p. 23, Plenum, New York, 1975.

Haerendel, G., G. Paschmann, N. Sckopke, H. Rosenbauer, and P. G. Hedgecock, The frontside boundary layer of the magnetosphere and the problem of reconnection, J. Geophys. Res., 83, 3195, 1978.

Hau, L.-N., and B. U. Sonnerup, The structure of resistive-dispersive intermediate shocks, J. Geophys. Res., 95, 18,791, 1990.

Heikkila, W. J., and J. D. Winningham, Penetration of magnetosheath plasma to low altitudes through the dayside magnetospheric cusps, J. Geophys. Res., 76, 883, 1971.

Karimabadi, H., D. Krauss-Varban, and N. Omidi, Kinetic structure of intermediate shocks: Implications for the magnetopause, J. Geophys. Res., 100, 11,957, 1995.

Klimov, S., et al., ASPI experiment: Measurements of fields and waves onboard the Interball-1 spacecraft, Ann. Geophys., 15, 514, 1997.
Lee, L. C., Y. Lin, Y. Shi, and B. T. Tsurutani, Slow shock characteristics as a function of distance from the X-line in the magnetotail, Geophys. Res. Lett., 16, 903, 1989.

Lin, Y., and H. Xie, Formation of reconnection layer at the dayside magnetopause, Geophys. Res. Lett., 24, 3145, 1997.

Lockwood, M., and M. F. Smith, Low and middle altitude cusp signatures for general magnetopause reconnection rate variations, 1, Theory, J. Geophys. Res., 99, 8531, 1994.

Lundin, R., Plasma composition and flow characteristics in the magnetospheric boundary layers connected to the polar cusp, in The Polar Cusp, edited by J. A. Holter and A. Egeland, pp. 9-32, D. Reidel, Norwell, Mass., 1985.

Lundin, R., Acceleration/heating of plasma on auroral field lines: Preliminary results from the Viking satellite, Ann. Geophys., 6, 143, 1988.

Lyu, L.-H., and J. R. Kan, Structures of Alfven shocks: S-shaped magnetic hododram observed at the magnetopause, Geophys. Res. Lett., 16, 349, 1989.

Miura, A., Simulation of Kelvin-Helmholz instability at the magnetospheric boundary, J. Geophys. Res., 92, 3195, 1987.

Moore, T. E., et al., The Thermal Ion Dynamics Experiment and Plasma Source Instrument, Space Sci. Rev., 71, 409, 1995.

Nakamura, M., and M. Scholer, Structure of the magnetopause reconnection layer and of flux transfer events: Ion kinetic effects, J. Geophys. Res., $105,23,179,2000$.

Newell, P. T., and C.-I. Meng, The cusp and the cleft/boundary layer: Lowaltitude identification and statistical local time variation, J. Geophys. Res., 93, 14,549, 1988.

Omidi, N., and D. Winske, Structure of slow magnetosonic shocks inlow beta plasmas, Geophys. Res. Lett., 16, 907, 1989.

Omidi, N., and D. Winske, Kinetic structure of slow shocks: Effects of the electromagnetic ion/ion cyclotron instability, J. Geophys. Res., 97, 14,801, 1992.

Paschmann, G., G. Haerendel, N. Sckopke, H. Rosenbauer, and P. G. Hedgecock, Plasma and magnetic field characteristics of the distant polar cusp near local noon: The entry layer, J. Geophys. Res., 81, 2883, 1976.

Paschmann, G., et al., Plasma acceleration at the Earth's magnetopause: Evidence for reconnection, Nature, 282, 243, 1979.

Petrinec, C. M., P. Song, and C. T. Russell, Solar cycle variations in the size and shape of the magnetopause, J. Geophys. Res., 96, 7893, 1991.

Petschek, H. E., Magnetic field annihilation, in AAS-NASA Symposium of the Physics of Solar Flares, edited by W. H. Hess, NASA Spec. Publ., SP50, 425, 1964.

Puhl-Quinn, P. A., and J. D. Scudder, Systematics of ion Walen analysis of rotational discontinuities using E/Z measurements, J. Geophys. Res., 105, 7617,2000

Rosenbauer, H., H. Grunwald, M. D. Montgomery, G. Paschmann, and N. Sckopke, Heos-2 plasma observations in the distant polar magnetosphere: The plasma mantle, J. Geophys. Res., 80, 2723, 1975.

Russell, C. T., and R. Elphic, Initial ISSE magnetometer results: Magnetopause observations, Space Sci. Rev., 22, 681, 1978.

Russell, C. T., et al., The GGS/Polar magnetic fields investigation, in The Global Geospace Mission, edited by C. T. Russell, p. 497, Kluwer Acad., Norwell, Mass., 1995.

Russell, C. T., G. Le, and S. M. Petrinec, Cusp observations of high and low-latitude cusp reconnection for northward IMF: Analternate view, J. Geophys. Res., 105, 5489, 2000.

Sauvaud, J. A., et al., The Interball-Tail Electron experiment: Initial results on the low-latitude boundary layer of the dawn magnetosphere, Ann. Geophys., 15, 587, 1997.

Savin, S., et al., Interball tail probe measurements in outer cusp and boundary layers, in Encounter Between Global Observations and Models in the ISTP Era, edited by D. Gallaher, J. Horwitz, and T. Moore, AGU, Washington, D. C., 1998.

Scholer, M., Magnetic flux transfer at the magnetopause based on single X-line bursty reconnection, Geophys. Res. Lett., 15, 291, 1988.

Scholer, M., Asymmetric time-dependent and stationary magnetic reconnection at the dayside magnetopause, J. Geophys. Res., 94, 15,099, 1989.

Scudder, J., et al., A 3-dimensional electron and ion hot plasma instrument for the Polar spacecraft of GGS mission, Space Sci. Rev., 71, 459, 1995. Scudder, J. D., P. Puuhl-Quinn, F. S. Moser, K. Ogilvie, and C. T. Russell, Generalized Walen tests through Alfven waves and rotational discontinuities using electron flow velocities, J. Geophys. Res., 104, 19,817, 1999. Semenov, V. S., I. Kubyshkin, V. Lebedeva, M. Sidneva, H. K. Biernat, M. F. Heyn, B. Besser, and R. Rijnbeek, Time-dependent localized reconnection of skewed magnetic fields, J. Geophys. Res., 97, 4251, 1992.

Shue, J.-H., J. K. Chao, H. C. Fu, C. T. Russell, P. Song, K. Khurana, and H. Singer, A new functional form to study the solar wind control of magnetopause size and location, J. Geophys. Res., 102, 9497, 1997.

Sibeck, D. G., Transient events in the outer magnetosphere, boundary waves or flux transfer events, J. Geophys. Res., 97, 4009, 1992. 
Smith, M. F., and M. Lockwood, Earth's magnetospheric cusps, Rev. Geophys., 34, 233, 1996.

Song, P., C. T. Russell, and M. F. Thomsen, Slow mode transition in the frontside magnetosheath, J. Geophys. Res., 97, 8225, 1992.

Song, P., B. U. O. Sonnerup, and M. F. Thomsen (Eds.), Physics of the Magnetopause, Geophys. Monogr. Ser., vol. 90, 447 pp., AGU, Washington, D. C., 1995.

Song, P., C. T. Russell, T. I. Gombosi, J. R. Spreiter, S. S. Stahara, and X. X. Zhang, On the processes in the terrestrial magnetosheath, 1, Scheme development, J. Geophys. Res., 104, 22,345, 1999a.

Song, P., C. T. Russell, X. X. Zhang, S. S. Stahara, J. R. Spreiter, and T. I. Gombosi, On the processes in the terrestrial magnetosheath, 2, Case study, J. Geophys. Res., 104, 22,357, 1999 b.

Song, P., T. I. Gombosi, D. L. DeZeeuw, K. G. Powell, and C. P. T. Groth, A model of solar wind-magnetosphere-ionosphere coupling for due northward IMF, Planet. Space Sci., 48, 29, 2000.

Sonnerup, B. U., et al., Evidence for magnetic field reconnection at the Earth's magnetopause, J. Geophys. Res., 86, 10,049, 1981

Southwood, D. J., C. J. Farrugia, and M. A. Saunders, What are flux transfer events?, Planet. Space Sci., 36, 503, 1988.

Spreiter, J. R., and S. S. Stahara, A new predictive model for determining solar wind-terrestrial planet interaction, J. Geophys. Res., 85, 6769, 1980.

Tsyganenko, N. A., Modeling the Earth's magnetospheric magnetic field confined within a realistic magnetopause, J. Geophys. Res., 100, 5599, 1995.

Vaisberg, O. L., et al., Initial observations of fine structures at the flank magnetopause with the complex plasma analyzer SCA-1 onboard the Interball Tail probe, Ann. Geophys., 15, 570, 1997.
Yermolaev, Y., et al., Ion distribution dynamics near the Earth's bow shock: First measurements with the 2D ion energy spectrometer CORALL on the INTERBALL/Tail satellite, Ann. Geophys., 15, 533, 1997.

L. Avanov, A. Fedorov, S. Savin, and A. Skalsky, Space Research Institute, 84/32 Profsoyuznaya Street, 117810 Moscow, Russia. (af@afed. iki.rssi.ru; sms7@po.cwru.edu)

M. O. Chandler, Space Science Laboratory, NASA Space Flight Center, Mail Stop ES-83, Huntsville, AL 35812, USA. (michael.chandler@) msfc.nasa.gov)

E. Dubinin, Max-Planck Institut für Aeronomie, Max-Planck-Strasse 2, D-37191 Katlenburg-Lindau, Germany. (dubinin@linmpi.mpg.de)

R. H. W. Friedel, Los Alamos National Laboratory, NIS-2, Mail Stop D-436, Los Alamos, NM 87545, USA. (friedel@nis.lanl.gov)

J. Kozyra and P. Song, Space Physics Research Laboratory, 2455 Hayward Street, Ann Arbor, MI 48109-2143, USA. (jukozyra@engin. umich.edu; psong@engin.umich.edu)

T. E. Moore, Laboratory for Extraterrestrial Physics, Goddard Space Flight Center, Code 692, Building 2, Room 138, Greenbelt, MD 20771 1000, USA. (thomas.e.moore@gsfc.nasa.gov)

C. T. Russell, Institute of Geophysics and Planetary Physics, University of California, Los Angeles, 3845 Slichter Hall, 405 Hilgard Avenue, Los Angeles, CA 90095-1567, USA. (ctrussel@igpp.ucla.edu)

J.-A. Sauvaud, CESR-CNRS, 9 avenue du Colonel Roche, PBP 4346, 31028 Toulouse cedex 4, France. (sauvaud@cesr.fr) 


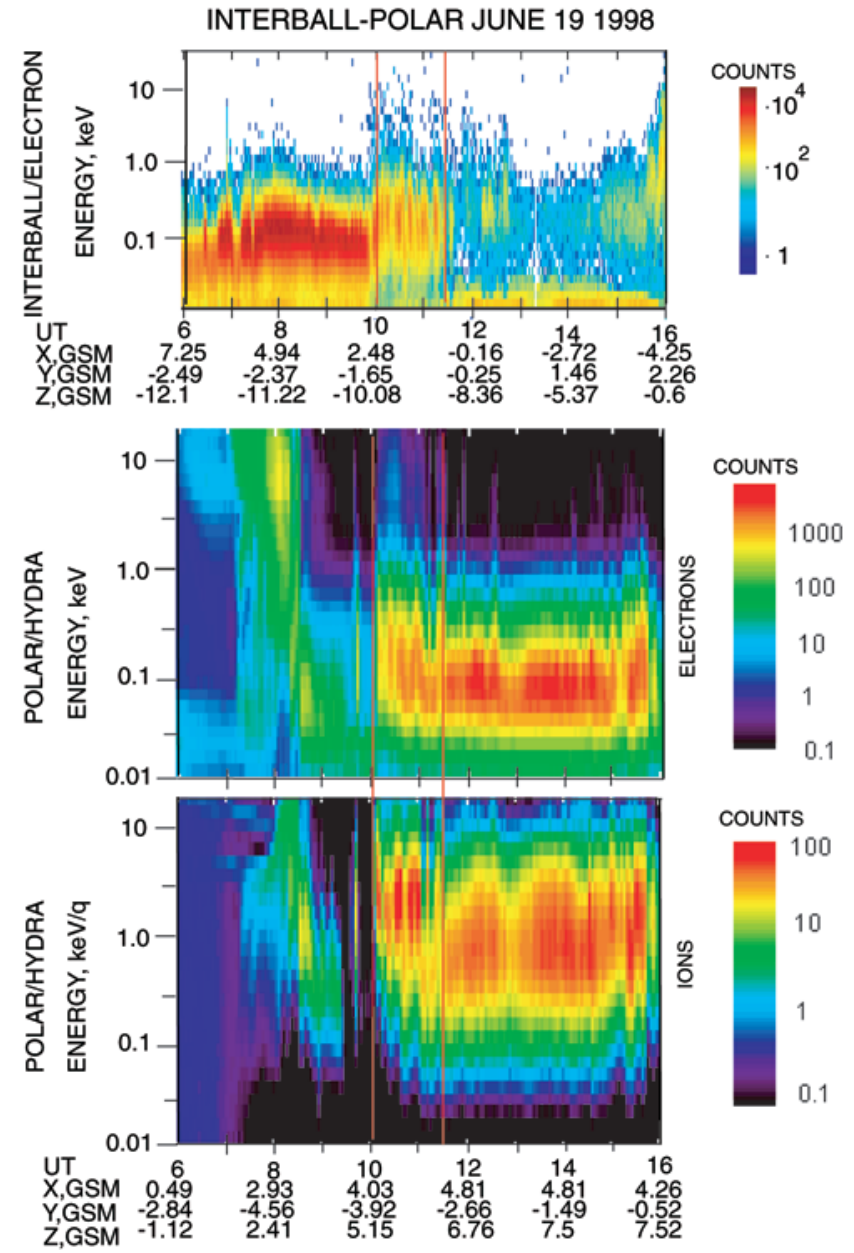

Figure 2. (top) Spectrogram of electron fluxes measured by Interball and spectrograms of (middle) electron fluxes and (bottom) ion fluxes measured by the HYDRA instrument on board Polar are displayed. Red vertical lines bound the time interval when Interball and Polar made coordinated measurements in the southern and northern high-latitude boundary layers close to the cusps. 
19 JUNE 1998

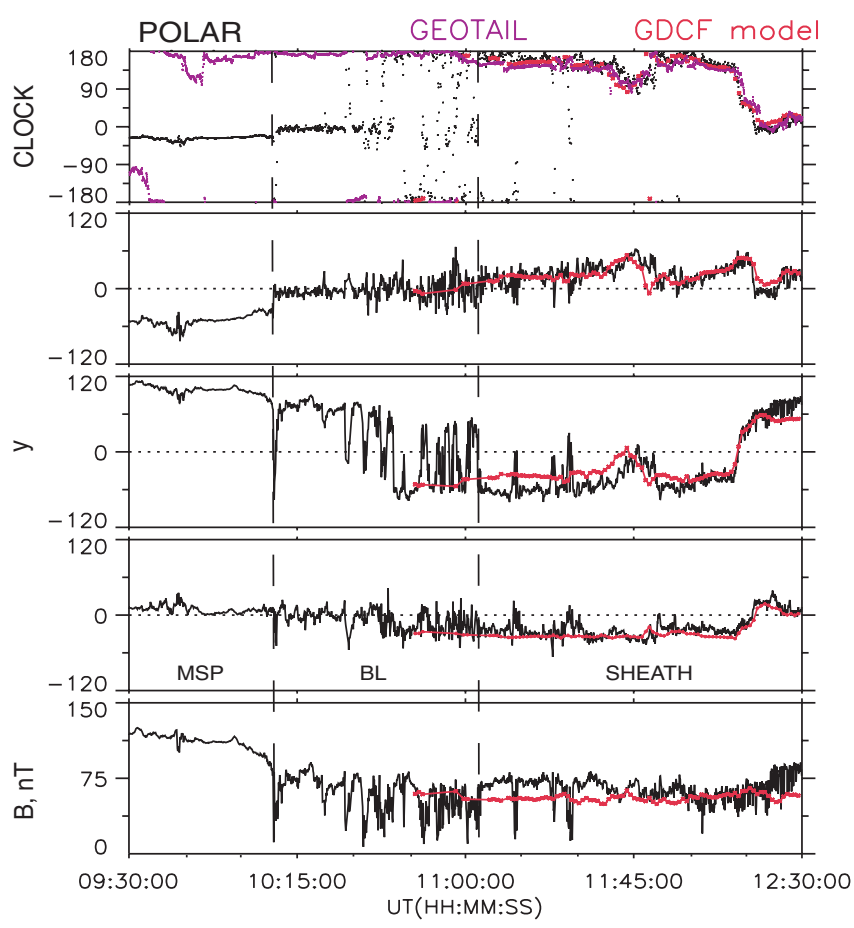

19 JUNE 1998

GEOTAIL

GDCF model

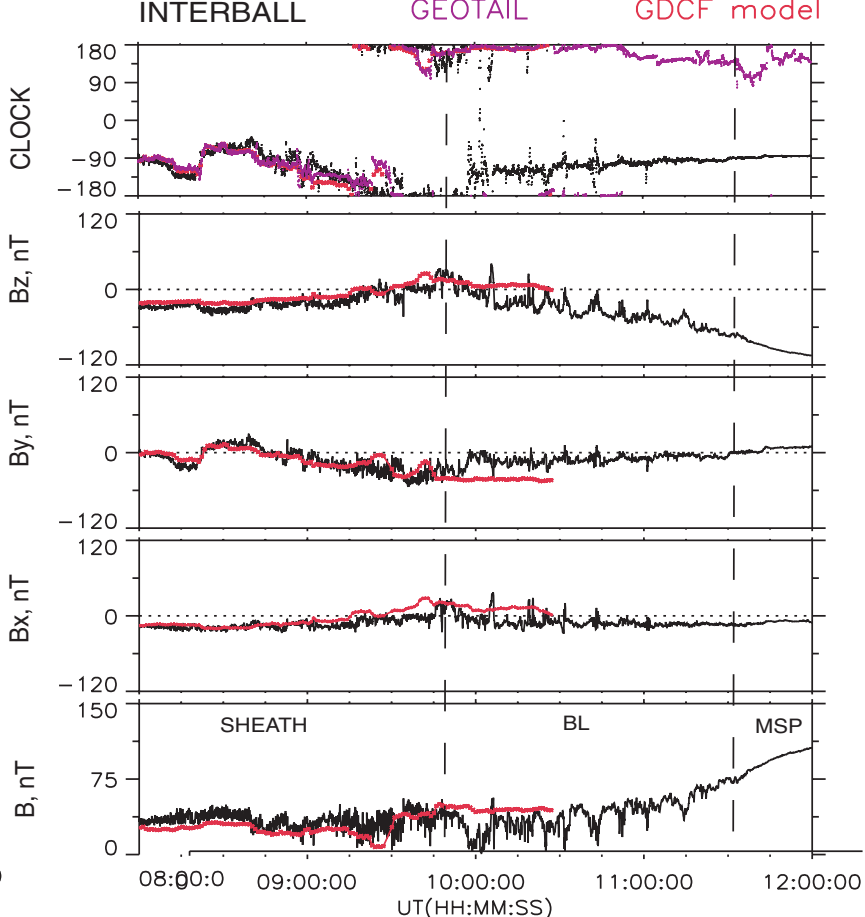

Figure 3. Comparison between the predictions from the gas dynamic model based on the plasma and the magnetic field measurements in the solar wind made by Geotail (red lines) with in situ measurements made by Polar and Interball (black lines). From top to bottom are given the clock angle, three components of the magnetic field in the GSE coordinates, and the magnetic field strength. The clock angle in the IMF with time shift of 10 min to account for the convection time from Geotail to Polar/Interball is plotted by a raspberry line. 

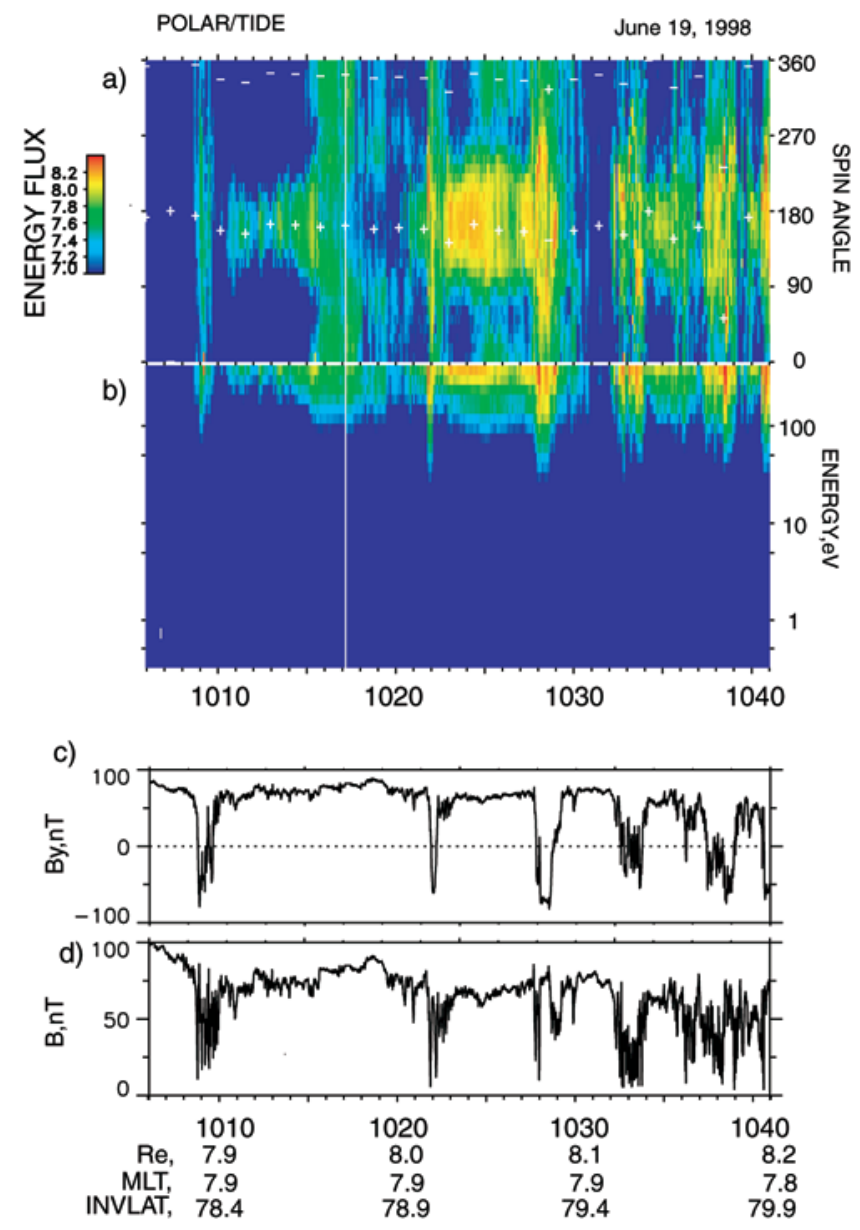

Figure 5. (a) Angular distribution of ion energy fluxes measured by TIDE in the boundary layer. The direction of the spin-averaged magnetic field is marked by pluses. The antiparallel direction is indicated by minus signs. The energy flux of ions is coded by color. (b) Energy-time spectrogram of ion fluxes. (c and d) Variations in the $B_{y}$ component and the magnetic field strength, given for reference. 

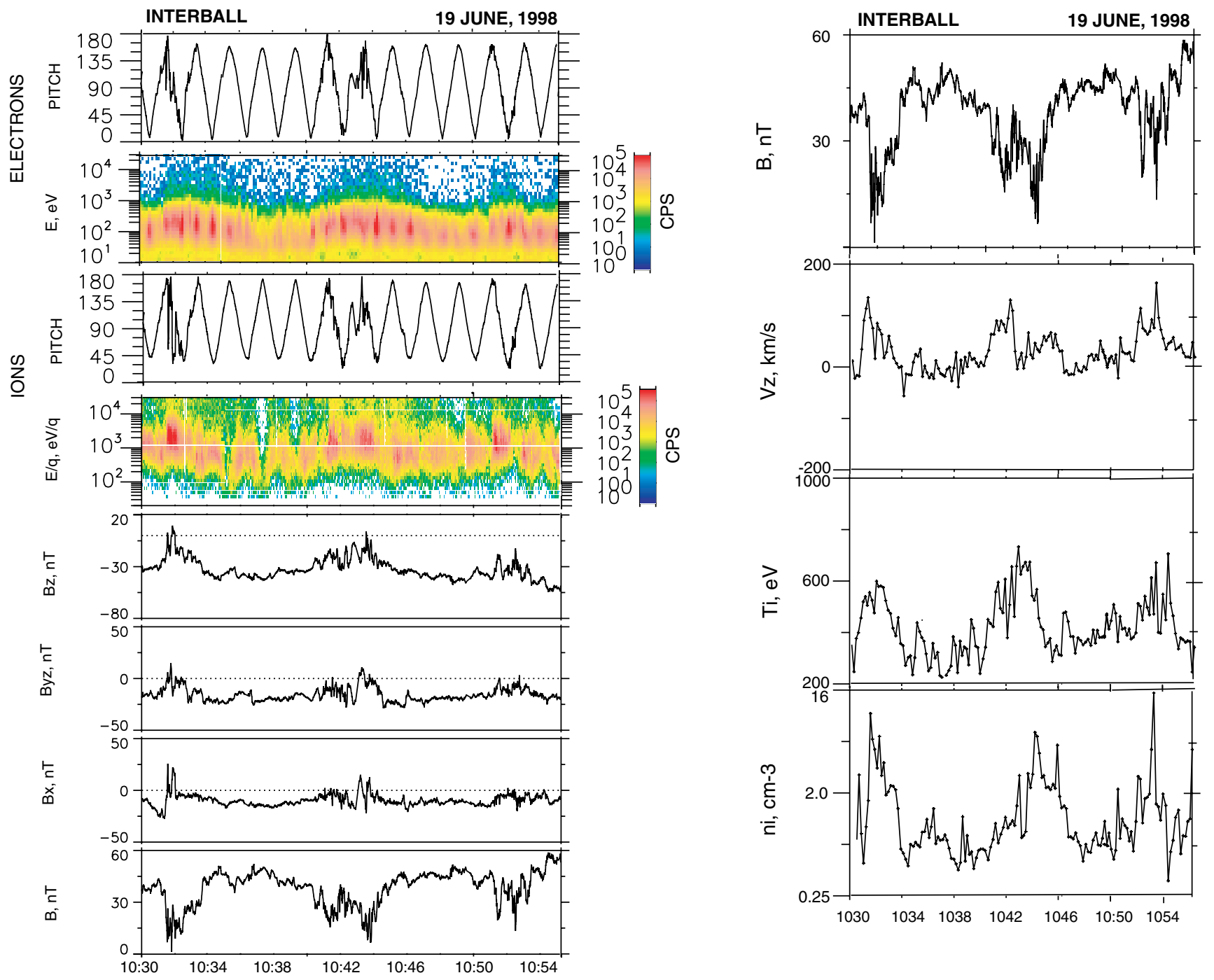

Figure 6. The fine structure of the events observed by Interball is shown in the particle and field measurements. On the left, energy-time spectrograms of ions and electrons, pitch angles of particles, and the magnetic field variations are given. On the right the plasma moments, namely, the component $V_{z}$ (GSE) of the bulk speed, the ion temperature, and the density, are displayed. The top right panel shows the magnetic field strength for reference. 

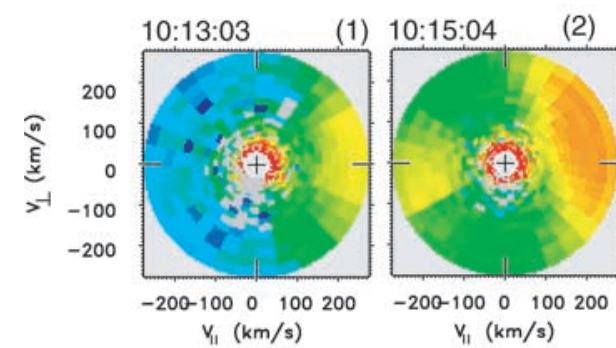

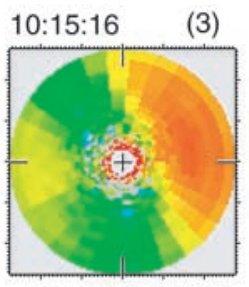

$-200-1000100200$

$V_{11}(\mathrm{~km} / \mathrm{s})$

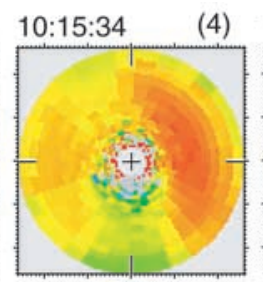

$-200-1000100200$

$V_{11}(\mathrm{~km} / \mathrm{s})$

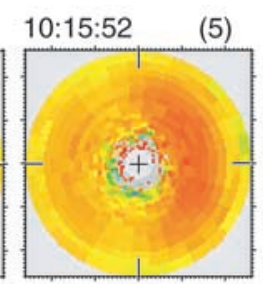

$-200-1000100200$

$V_{11}(\mathrm{~km} / \mathrm{s})$

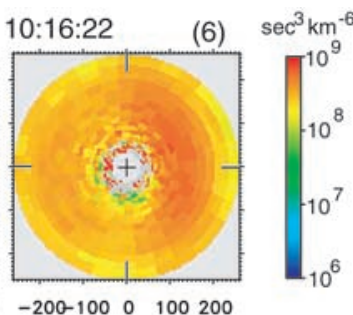

$V_{11}(\mathrm{~km} / \mathrm{s})$
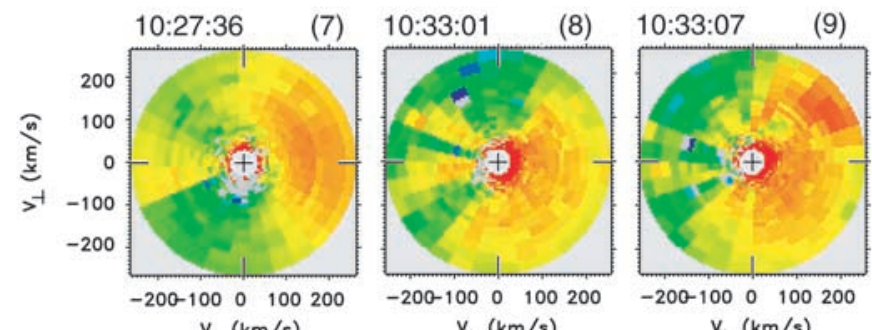

$V_{11}(\mathrm{~km} / \mathrm{s})$

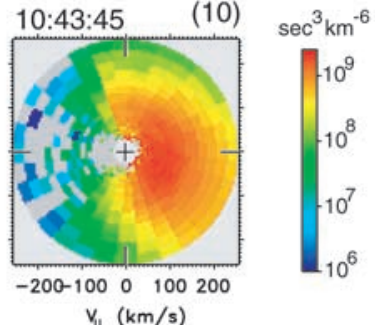

Figure 10. A set of 2-D velocity distributions of ions in $V_{\|} V_{\perp}$ coordinates.

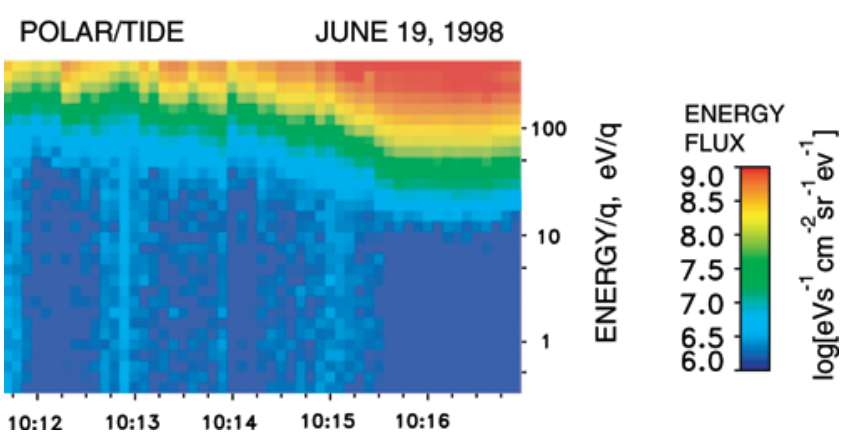

Figure 12. Energy-time spectrogram of field-aligned ions measured when Polar observed the reconnection event at 1012-1017 UT. The value of the low-energy cutoff of injected ions carries information about the distances from the reconnection sites. 